ARTICLE

\title{
Distinct roles of KLF4 in mesenchymal cell subtypes during lung fibrogenesis
}

\author{
Rachana R. Chandran 1,2, Yi Xie (1) 1,3, Eunate Gallardo-Vara (1) 1,2, Taylor Adams ${ }^{4}$, Rolando Garcia-Milian (i) 5 , \\ Inamul Kabir (1) 1,2, Abdul Q. Sheikh¹,7, Naftali Kaminski (1) 4, Kathleen A. Martin,3, Erica L. Herzog ${ }^{4,6}$ \& \\ Daniel M. Greif (10 1,2凶
}

During lung fibrosis, the epithelium induces signaling to underlying mesenchyme to generate excess myofibroblasts and extracellular matrix; herein, we focus on signaling in the mesenchyme. Our studies indicate that platelet-derived growth factor receptor (PDGFR)- $\beta^{+}$ cells are the predominant source of myofibroblasts and Kruppel-like factor (KLF) 4 is upregulated in PDGFR- $\beta^{+}$cells, inducing TGF $\beta$ pathway signaling and fibrosis. In fibrotic lung patches, KLF4 is down-regulated, suggesting KLF4 levels decrease as PDGFR- $\beta^{+}$cells transition into myofibroblasts. In contrast to PDGFR- $\beta^{+}$cells, KLF4 reduction in $\alpha$-smooth muscle actin $(\mathrm{SMA})^{+}$cells non-cell autonomously exacerbates lung fibrosis by inducing macrophage accumulation and pro-fibrotic effects of PDGFR- $\beta^{+}$cells via a Forkhead box M1 to C-C chemokine ligand 2 -receptor 2 pathway. Taken together, in the context of lung fibrosis, our results indicate that KLF4 plays opposing roles in PDGFR- $\beta^{+}$cells and SMA+ cells and highlight the importance of further studies of interactions between distinct mesenchymal cell types.

\footnotetext{
${ }^{1}$ Yale Cardiovascular Research Center, Section of Cardiovascular Medicine, Department of Internal Medicine, Yale University School of Medicine, New Haven, CT 06511, USA. ${ }^{2}$ Department of Genetics, Yale University School of Medicine, New Haven, CT 06520, USA. ${ }^{3}$ Department of Pharmacology, Yale University School of Medicine, New Haven, CT 06520, USA. ${ }^{4}$ Section of Pulmonary, Critical Care and Sleep Medicine, Department of Internal Medicine, Yale University School of Medicine, New Haven, CT 06520, USA. ${ }^{5}$ Bioinformatics Support Program, Yale University School of Medicine, New Haven, CT 06520,

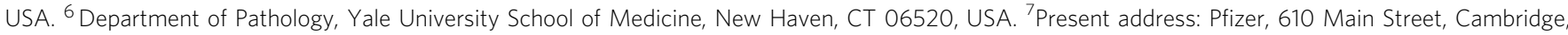
MA 02139, USA. ${ }^{凶}$ email: daniel.greif@yale.edu
} 
$\mathrm{P}$ ulmonary fibrosis is a devastating disease that results from insults to the lung and is characterized by the exaggerated production and impaired turnover of extracellular matrix (ECM) leading to architectural distortion and impaired gas transfer in the lung ${ }^{1}$. Pulmonary fibrosis has diverse known causes including exposure to toxins or bleomycin or is idiopathic (IPF). IPF, the most common form of interstitial lung disease, is particularly lethal causing 40,000 deaths annually in the USA alone, and the median survival after initial diagnosis is a dismal $\sim 3$ years $^{2,3}$. The course of IPF is highly variable as for unclear reasons, some patients experience rapid deterioration in lung function while others experience more gradual decline ${ }^{4,5}$. The success of two drugs, pirfenidone and nintedanib, in delaying disease progression indicates that the clinical course can be altered, but the benefit of these agents is unpredictable, at best modest, and accompanied by significant toxicities ${ }^{2,6}$.

These major limitations in prognostication and treatment options for pulmonary fibrosis largely stem from our inadequate understanding of the underlying cellular and molecular mechanisms driving pathogenesis. In the normal lung, $\alpha$-smooth muscle actin (SMA) is expressed by airway and vascular smooth muscle cells (SMCs) and the very rare alveolar myofibroblasts ${ }^{7}$. A hallmark of pulmonary fibrosis is a marked increase in myofibroblasts which are implicated in pathological contraction of lung tissue and may contribute to synthesis of excessive $\mathrm{ECM}^{2,3,8}$. The current paradigm of IPF pathogenesis suggests that repeated alveolar epithelial cell injury activates the underlying mesenchyme and results in accumulation of excess myofibroblasts and ECM through as yet undefined mechanisms ${ }^{9,10}$. This paradigm points towards signaling from the epithelium to the mesenchyme; however, in the context of lung fibrosis, little attention has been paid to the crosstalk between mesenchymal cell types (e.g., signaling between SMCs and fibroblasts). In addition, the role of SMCs in general during fibrosis pathogenesis is not well understood.

The cellular origins of myofibroblasts in lung fibrosis are incompletely defined although multiple postembryonic mesenchymal cell populations are believed to be dominant sources. Relevant studies have relied heavily on the bleomycin mouse model of lung fibrosis which shares key histopathological findings with human IPF, including excessive and aberrant myofibroblast accumulation and collagen deposition and obliteration of alveoli ${ }^{11,12}$. In the context of bleomycin exposure, fate mapping studies have implicated $\mathrm{ADRP}^{+}$lipofibroblasts ${ }^{13}$, Axin $2^{+}$progenitors ${ }^{14}, \mathrm{Gli1}^{+}$mesenchymal stem cell-like cells ${ }^{15}$ and FoxD $1^{+}$progenitor-derived pericytes ${ }^{16}$ as sources of substantial myofibroblasts whereas another study indicates that "pericyte-like cells" expressing NG2 or FoxJ1 do not contribute ${ }^{17}$. The mesenchyme marker platelet-derived growth factor receptor (PDGFR) $\beta$ is expressed by many of these mesenchymal cell types, including fibroblasts, pericytes, $\mathrm{Gli1}^{+}$mesenchymal stem cell (MSC)-like cells and Axin2 ${ }^{+}$progenitors ${ }^{14,15,18}$, but no prior studies have utilized cell labeling driven by an inducible Pdgfrb promoter for fate mapping in lung fibrosis.

Beyond cellular origins, insights into molecular pathways critical for mesenchymal cell transitions during the pathogenesis of lung fibrosis are needed. Treatment with an anti-PDGFR- $\beta$ blocking antibody has been shown to attenuate bleomycininduced lung fibrosis and proliferation of cells in the interalveolar space ${ }^{19}$. Herein, we focus on Kruppel-like factor 4 (KLF4), a pluripotency transcription factor (TF) integral in cell fate and reprogramming ${ }^{20-22}$. KLF4 is expressed in diverse cell types found in the lung, including epithelial cells, leukocytes and fibroblasts as well as in the context of disease, in perivascular cells (i.e., SMCs and/or pericytes) ${ }^{23-26}$. Myofibroblasts express SMC markers (e.g., SMA), and in SMCs, studies indicate that KLF4 induces dedifferentiation of $\mathrm{SMCs}^{27,28}$; in contrast, however, there is no consensus regarding the role of KLF4 in myofibroblast differentiation and fibrosis. For instance, in vimentin ${ }^{+}$cells isolated from neonatal mouse hearts and cultured in conditions to eliminate cardiomyocytes, KLF4 induces SMA levels and potentiates angiotensin II-induced collagen levels ${ }^{29}$; however, KLF4 function in renal fibrosis is controversial with studies suggesting that KLF4 is either protective or deleterious ${ }^{30}$. In the lung, KLF4 has been implicated in the normal perinatal accumulation of myofibroblasts as $K l f 4^{(-/-)}$neonates lack alveolar myofibroblasts, and expression of KLF4 in lung fibroblasts isolated from Klf4 null mice induces expression of SMA, collagen and fibronectin ${ }^{23}$. In contrast, a study of cultured cells with fibroblast-like morphology isolated from the rodent lung reported that KLF4 binds SMAD3 and thereby, inhibits activation of the Acta2 promoter $^{31,32}$. Finally, a recent study reported that KLF4 overexpression by unspecified cells in the mouse attenuates bleomycin-induced lung fibrosis ${ }^{33}$.

Herein, we use conditional genetic labeling to demonstrate that select PDGFR- $\beta^{+}$cells clonally expand and give rise to the majority of myofibroblasts in bleomycin-induced lung fibrosis whereas only rare myofibroblasts derive from preexisting $\mathrm{SMA}^{+}$ cells. Furthermore, these studies delineate mesenchymal cell-typespecific roles of KLF4 in lung fibrosis. KLF4 expression is upregulated in PDGFR- $\beta^{+}$cells and their lineage during disease pathogenesis. Reduction of KLF4 in PDGFR- $\beta^{+}$cells attenuates fibrosis, at least in part by downregulating transforming growth factor (TGF) $\beta$ pathway activation and thereby, reducing ECM levels. In stark contrast, $\mathrm{Klf} 4$ deletion in $\mathrm{SMA}^{+}$cells exacerbates fibrosis, and the data indicate that this effect is largely noncell autonomous. Indeed, decrease of KLF4 levels in SMA ${ }^{+}$cells enhances $\mathrm{C}-\mathrm{C}$ motif chemokine ligand 2 (CCL2) levels via Forkhead box M1 (FOXM1) upregulation, induces macrophage recruitment and stimulates profibrotic transformation of PDGFR$\beta^{+}$cells via the cognate receptor $\mathrm{C}-\mathrm{C}$ motif chemokine receptor 2 (CCR2). Thus, KLF4 plays critical and contrasting roles in the regulation of lung fibrosis depending on the specific mesenchymal cell type: profibrotic in PDGFR- $\beta^{+}$cells and antifibrotic in $\mathrm{SMA}^{+}$cells.

\section{Results}

PDGFR- $\beta^{+}$cells are the primary source of lung myofibroblasts. The normal adult lung has only very rare $\mathrm{SMA}^{+}$ myofibroblasts $7,34,35$, but this cell type accumulates in the fibrotic lung and is considered to play key pathological roles. PDGFR- $\beta$ is expressed in diverse cell types, including fibroblasts, pericytes, Axin2 ${ }^{+}$myofibrogenic progenitors, Gli1 ${ }^{+}$MSC-like cells and select vascular SMCs, of which all except the latter have been implicated as a source of lung myofibroblasts $7,14,15,18,24$. Almost all $\mathrm{SMA}^{+}$lung myofibroblasts in bleomycin-induced fibrosis express PDGFR- $\beta^{19}$ (Supplementary Fig. 1), and thus, studies with the constitutive Pdgfrb-Cre in this context are not informative with regard to cellular origins of myofibroblasts ${ }^{36}$. No prior studies have conditionally labeled PDGFR- $\beta^{+}$cells (with for instance, $P d g f r b-C r e E R^{T 2}$ ) to follow their fate during the course of lung fibrosis. To initially assess what cell types in the lung are marked by $P d g f r b-C r e E R^{T 2}$ under basal conditions, PdgfrbCreER $R^{T 2}$, ROSA26R ${ }^{(\mathrm{Zs} /+)}$ mice were induced with tamoxifen ( $1 \mathrm{mg} /$ day for 5 days) and then rested for 5 days (Supplementary Fig. 2). The lungs were subjected to $10 \mathrm{X}$ Genomics single-cell (sc) RNA library construction followed by sequencing (seq), and scRNA-seq data were aligned to a modified version of the mouse transcriptome mm10, including the ZsGreen1 (Zs) sequence. Cells were annotated and clustered in concordance with a recent scRNA-seq analysis of collagen-producing lung cells ${ }^{37}$, and a 
uniform manifold approximation and projection was used to visualize Zs and Pdgfrb expression in lung stromal cells (Supplementary Figs. 3a-c and 4). These results suggest that both Pdgfrb and $Z s$ are expressed in diverse populations of fibroblasts as well as pericytes in the $P d g f r b-C r e E R^{T 2}, R O S A 26 R^{(Z s /+)}$ lung. A previous scRNA-seq analysis demonstrates Pdgfrb expression in multiple similar lung fibroblast populations ${ }^{38}$.

In addition, the expression patterns of Gli1, Pdgfra and Axin2 were evaluated (Supplementary Fig. 3d-f). Prior immunohistochemical analysis indicates that Gli1 ${ }^{+}$cells express PDGFR- $\alpha$ and PDGFR- $\beta^{15}$, and our scRNA-seq shows that both Glil and Pdgfra are primarily expressed in adventitial and alveolar fibroblasts, clusters that are largely Pdgfrb ${ }^{+}$. In contrast, consistent with prior studies $^{14,37}$, Axin2 is broadly expressed. Peribronchiolar Axin2 ${ }^{+}$ cells that are predominantly PDGFR- $\alpha^{-}$PDGFR- $\beta^{+}$are implicated as myofibrogenic progenitor cells, and these Axin $2^{+}$cells have relatively high mRNA levels of Acta2, transgelin and desmin ${ }^{14}$. Based on this expression pattern and our scRNA-seq data (Supplementary Fig. 3b, e-i), Axin $2^{+}$myofibrogenic progenitors may be enriched in our peribronchial fibroblast/SMC cluster.

We then proceeded to fate mapping of PDGFR- $\beta^{+}$cells during lung fibrosis. To this end, Pdgfrb-CreER $\left.{ }^{T 2}, \operatorname{ROSA}_{2} 6 R^{(m T m G /+}\right)$ mice were injected with tamoxifen and rested to mark PDGFR- $\beta^{+}$ cells (Supplementary Fig. 5a, b) and then treated with a single orotracheal dose of phosphate-buffered saline (PBS) or bleomycin to induce lung fibrosis (Fig. 1a and Supplementary Fig. 5c, d). In order to ensure lung myofibroblast accumulation, mice were analyzed at 7 and 14 days following bleomycin administration (the latter is a time point of established fibrosis). Of the total lung myofibroblasts, PDGFR- $\beta^{+}$cells give rise to $84 \pm 5 \%$ on day 7 following bleomycin administration and $68 \pm 9 \%$ on day 14 (Fig. 1a, c). In contrast, fate mapping with Acta2-CreER ${ }^{T 2}$, which in the normal lung marks airway and vascular SMCs as well as the very rare alveolar myofibroblasts, indicates that only $10 \pm 2 \%$ of bleomycin-induced myofibroblasts derive from preexisting $\mathrm{SMA}^{+}$ cells (Fig. 1b, c and Supplementary Fig. 6). Interestingly, in mice exposed to hypoxia as an alternate model of lung pathology that induces myofibroblast accumulation-similar to results with bleomycin-PDGFR- $\beta^{+}$cells give rise to a substantial percentage of myofibroblasts $(43 \pm 6 \%)$ (Supplementary Fig. 7a) whereas $\mathrm{SMA}^{+}$cells only contribute $\sim 10 \% 7$. To complement and extend the immunohistochemical studies in lung fibrosis, following bleomycin treatment of tamoxifen-induced Pdgfrb-CreER ${ }^{T 2}$, $\operatorname{ROSA} 26 R^{(\mathrm{Zs} /+)}$ mice, lung $\mathrm{Zs}^{+}$cells were isolated by fluorescence-activated cell sorting (FACS) and subjected to qRT-PCR. During lung fibrosis, the expression of Acta2 and collagen 1a1 (Colla1) and 3a1 (Col3a1) in the PDGFR- $\beta^{+}$cell lineage is markedly increased (Fig. 1d).

PDGFR- $\beta^{+}$cells proliferate and clonally expand during injury. Given that PDGFR- $\beta^{+}$cells are the main source of myofibroblasts in lung fibrosis, we next determined whether bleomycin induces PDGFR- $\beta^{+}$cell proliferation and if so, clonal expansion. Pdgfrb$C r e E R^{T 2}$, ROSA26R(Zs/+) mice were induced with tamoxifen, rested and then treated with a single dose of orotracheal PBS (control) or bleomycin. Fourteen days later, 5-ethynyl-2'-deoxyuridine (EdU) was administrated $12 \mathrm{~h}$ prior to euthanasia. Lung sections were stained for EdU and nuclei (DAPI) and directly imaged for Zs. Following bleomycin administration, $9.9 \pm 0.8 \%$ of the PDGFR- $\beta^{+}$cell lineage is EdU ${ }^{+}$in comparison to $0.7 \pm 0.3 \%$ following PBS (Fig. 1e, f).

To examine the clonality and mixing of the PDGFR- $\beta^{+}$cell lineage in the fibrotic lung, Pdgfrb-CreER ${ }^{T 2}$ mice carrying an allele of the multicolor Rainbow ( $\mathrm{Rb}$ ) Cre reporter, which upon recombination expresses Cerulean, mCherry or mOrange in a mutually exclusive manner 24,39 , were used. These mice were induced with tamoxifen, rested and then a single dose of orotracheal PBS or bleomycin was administered. At 14 days, the size of clonal patches in the lung parenchyma was scored based on contiguous cells labeled with the same fluorophore and then averaged or binned into patch size groups (Fig. 1g-i; see "Methods"). For mice treated with PBS or bleomycin, the mean patch size is $1.2 \pm 0.1$ or $7.0 \pm 1.7$ cells and the maximum patch size is 3 or 30 cells, respectively. Thus, upon bleomycin exposure, PDGFR- $\beta^{+}$cells undergo clonal expansion to form sizable congruent patches of cells that display limited mixing with other cells (Supplementary Fig. 8).

KLF4 is upregulated in PDGFR- $\beta^{+}$cell lineage during lung injury. We and others have studied the pluripotency factor KLF4 in the context of diverse relevant diseases, including pulmonary hypertension $^{7,24}$, lung cancer ${ }^{40}$ and organ fibrosis $29,30,33$; however, the role of KLF4 in PDGFR- $\beta^{+}$cells and cells derived from PDGFR- $\beta^{+}$cells in fibrosis has not been investigated. Our scRNA-seq indicates that Klf4 is broadly expressed in the normal lung under basal conditions, including in Pdgfrb ${ }^{+}$cells, and within the population of Pdgfrb ${ }^{+}$cells, Klf4 is most highly expressed in the adventitial fibroblast cluster (Supplementary Fig. 3j). We next evaluated KLF4 expression in PDGFR- $\beta^{+}$cells during the course of fibrosis at day 5 (in the acute inflammation phase $^{11}$ ) and at day 14 . In bleomycin-treated wild-type mice, lung sections demonstrate a $\sim 4$-fold increase in the percent of PDGFR$\beta^{+}$cells expressing KLF4 compared to untreated controls (Fig. 2a, b). Next, Pdgfrb-CreER ${ }^{T 2}$, ROSA26R ${ }^{(m T m G /+)}$ mice were induced with tamoxifen, rested and then treated with a single orotracheal dose of PBS (control) or bleomycin. Fourteen or twenty-one days later, mice were euthanized and lungs were harvested, sectioned and stained for GFP, KLF4 and nuclei (DAPI). Compared to control, lung sections from bleomycin-treated mice demonstrate a $\sim 3.5$-fold increase in the percent of $\mathrm{GFP}^{+}$cells expressing KLF4 at day 14 but no change at day 21 (Fig. 2c, d and Supplementary Fig. $9 \mathrm{a}, \mathrm{b}$ ). In addition to the substantial increase in the percent of PDGFR- $\beta^{+}$cells and PDGFR- $\beta^{+}$lineage cells expressing KLF4, the intensity of staining for KLF4 in these cells is markedly enhanced at day 14 after bleomycin (Fig. 2a, c). To further evaluate the expression level of Klf 4 in the PDGFR- $\beta^{+}$cell lineage, tamoxifen-induced $P d g f r b-C r e E R^{T 2}, R O S A 26 R^{(Z s /+)}$ mice were or were not treated with bleomycin. At 5, 14 or 21 days thereafter, lung $\mathrm{Zs}^{+}$cells were isolated by FACS and subjected to qRT-PCR for Klf4. In comparison to untreated mice, Klf4 transcript levels are increased by $\sim 2$-fold at day 5 and $\sim 3$-fold at day 14 and unchanged at day 21 (Fig. 2e and Supplementary Fig. 9c). In addition, we observed a marked reduction in KLF4 expression in $\mathrm{SMA}^{+}$fibrotic patches in the lungs of bleomycin-treated mice and of humans with IPF (Fig. 2f-i and Supplementary Fig. 10). Thus, KLF4 expression is increased in PDGFR- $\beta^{+}$cells and PDGFR- $\beta^{+}$lineage cells during bleomycin-induced fibrosis, and we speculate these cells downregulate KLF4 as they differentiate into $\mathrm{SMA}^{+}$cells in fibrotic patches of the lung.

Klf4 deletion in PDGFR- $\beta^{+}$cells mitigates pulmonary fibrosis. Given the early upregulation of KLF4 in PDGFR- $\beta^{+}$cells following bleomycin treatment, we hypothesized that KLF4 plays a role in the profibrotic transformation of PDGFR- $\beta^{+}$cells. To test

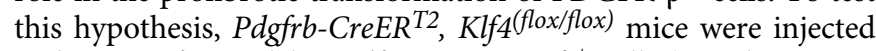
with tamoxifen to delete Klf4 in PDGFR- $\beta^{+}$cells (Supplementary Fig. 11a) or corn oil (vehicle), rested and subjected to a single orotracheal dose of bleomycin. Fourteen days later, mice were euthanized, and lungs were harvested and analyzed. Deletion of Klf4 in PDGFR- $\beta^{+}$cells considerably reduces bleomycin-induced 
14 days after bleomycin
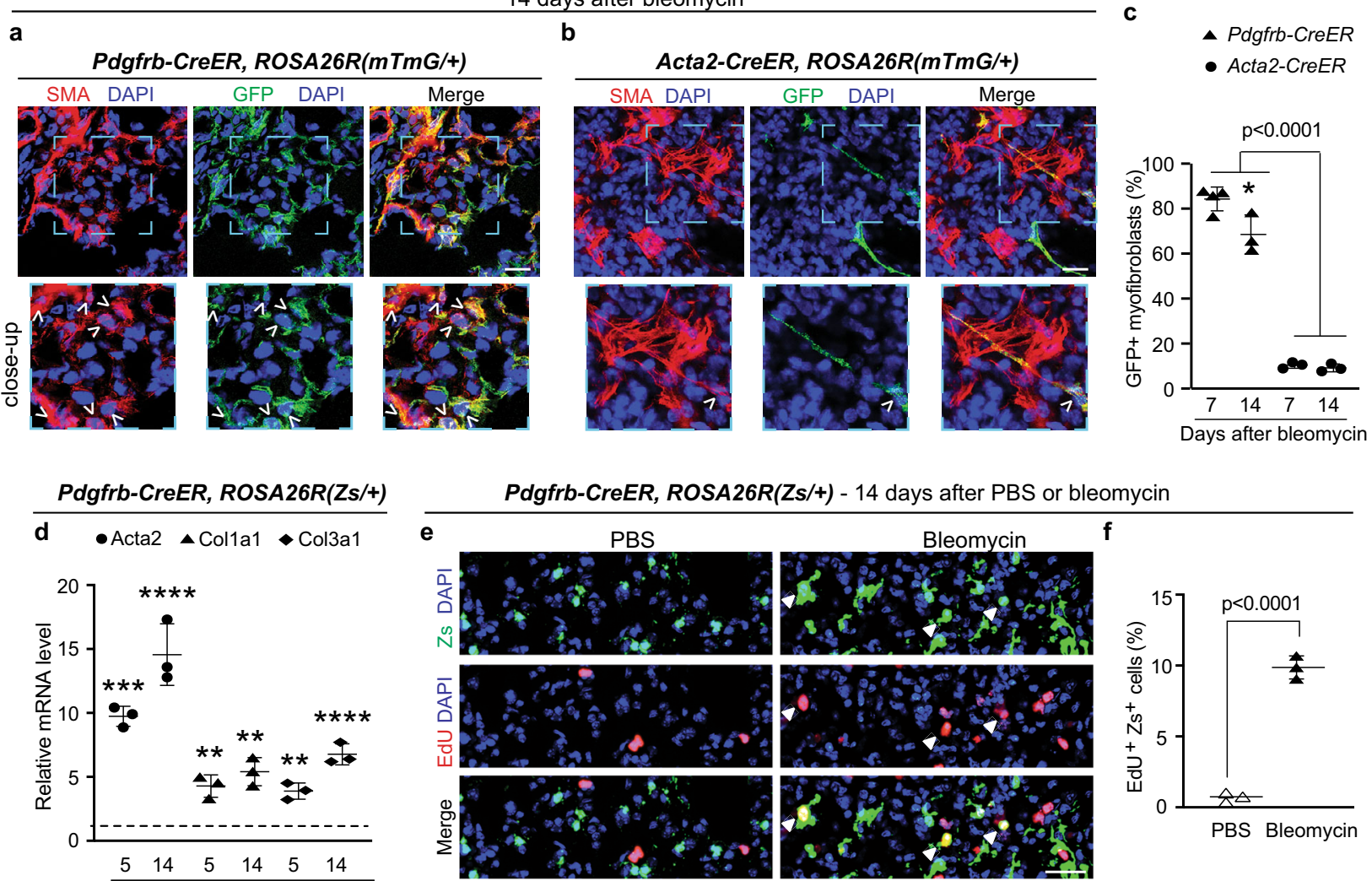

Pdgfrb-CreER, ROSA26R(Rb/+) - 14 days after PBS or bleomycin
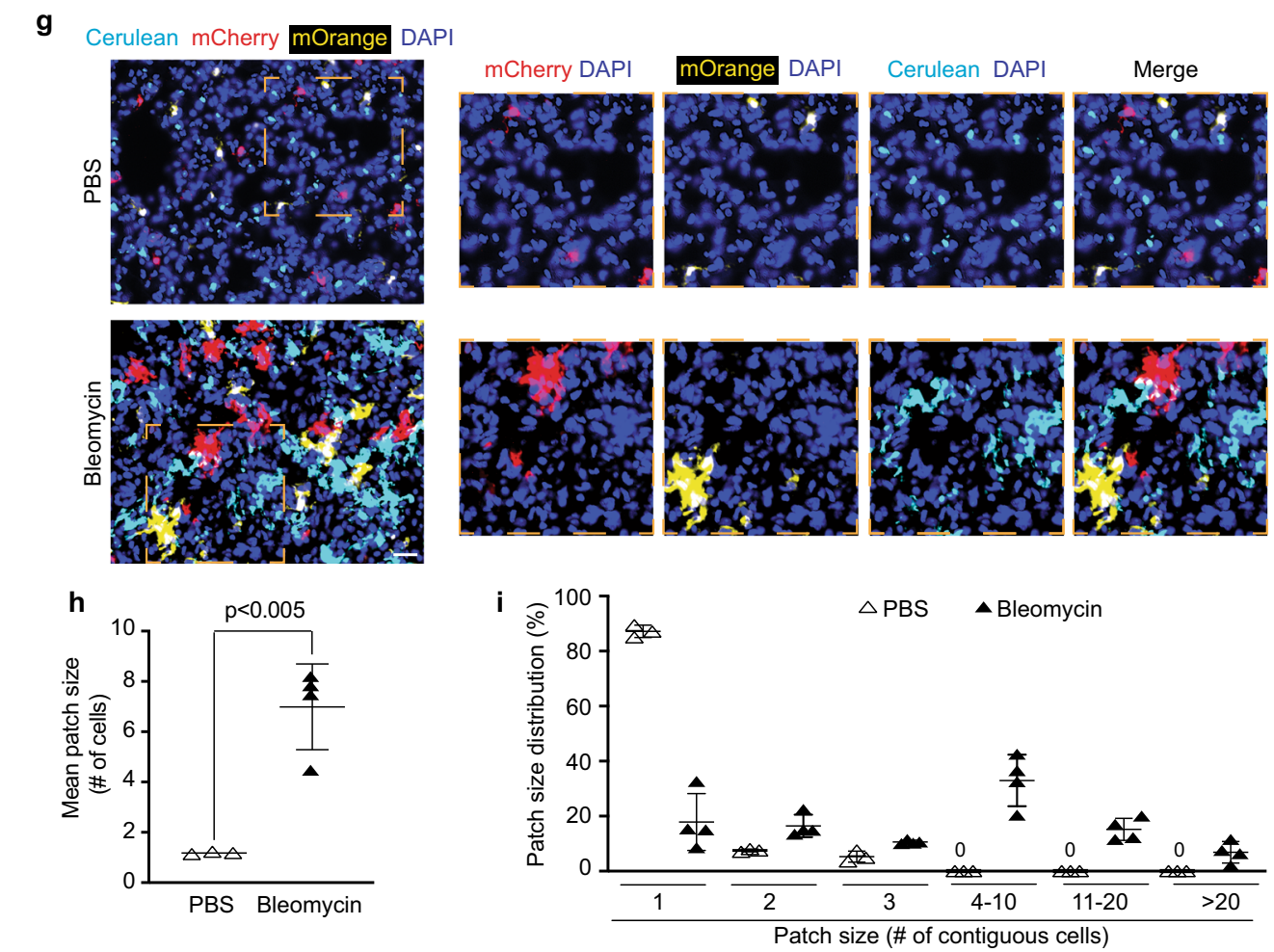

lung accumulation of excess $\mathrm{SMA}^{+}$myofibroblasts (Fig. 3a) and collagen as demonstrated by qualitative Picrosirius red staining (Fig. 3b) and the quantitative Sircol assay (Fig. 3c). Western blot analysis of whole lung lysates also revealed a reduction in collagen 1 (COL1), fibronectin 1 (FN1) and SMA protein levels (Fig. 3d, e). These data indicate that KLF4 expression in PDGFR- $\beta^{+}$cells is a key factor inducing myofibroblast accumulation and fibrosis in the lung upon bleomycin exposure. Once again, similar results were obtained with hypoxia as deletion of Klf4 in PDGFR- $\beta^{+}$cells prior to this exposure markedly reduces lung myofibroblasts (Supplementary Fig. 7b), thereby indicating the relevance of this pathway to multiple pathogenic processes in the lung. 
Fig. 1 PDGFR- $\beta^{+}$cells give rise to myofibroblasts, express collagen and clonally expand during lung fibrogenesis. a-c $R O S A 26 R^{(m T m G /+)}$ mice carrying Pdgfrb-CreER ${ }^{T 2}$ or Acta2-CreER ${ }^{T 2}$ were induced with tamoxifen, rested and subjected to an orotracheal bleomycin dose. Seven or fourteen days later, mice were euthanized, and lung vibratome sections were stained for SMA and GFP (fate marker). Images (a, b) with close-ups of boxed regions below. Arrowheads indicate GFP + myofibroblasts. Percent of myofibroblasts that are GFP + (c). For each genotype and time point, $n=3$ mice except $n=4$ mice for Pdgfrb-CreER ${ }^{T 2}$ at 7 days, 6-12 sections per mouse, average of 58 myofibroblasts per section; ${ }^{\star}$ vs. Pdgfrb-CreER ${ }^{T 2}$ at 7 days, $p=0.0143$. d PdgfrbCreER $R^{T 2}, \operatorname{ROSA} 26 R^{(\mathrm{Zs} /+)}$ mice were induced with tamoxifen, rested, treated or not treated with bleomycin, and 5 or 14 days later, $Z \mathrm{~s}^{+}$cells were isolated by FACS and subjected to GRT-PCR. Levels of indicated transcripts are relative to Gapdh and normalized to no treatment. For each treatment group and time point, $n=3$ mice in triplicate; ${ }^{\star \star}$, Col1a1, day $5(p=0.0071)$, Col1a1, day $14(p=0.0016)$, Col3a1, day $5(p=0.0027)$, ${ }^{\star \star \star} p=0.0008,{ }^{\star \star \star \star} p<0.0001$ vs. no treatment, respectively. e, f Pdgfrb-CreERT2, ROSA26R(Zs/+) were labeled with tamoxifen, rested and treated with PBS or bleomycin. On the evening of the 13th day thereafter, EdU was injected, and $12 \mathrm{~h}$ later, mice were euthanized. Lung cryosections were stained for EdU and directly imaged for Zs (e) with closed arrowheads indicating EdU ${ }^{+} \mathrm{Zs}^{+}$cells. Percent of $\mathrm{Zs}^{+}$cells that are EdU ${ }^{+}$is quantified (f). For each treatment group, $n=3$ mice, three sections per mouse, average $90 \mathrm{Zs}^{+}$cells per section. g-i Pdgfrb-CreER ${ }^{T 2}, R O S A 26 R^{(R b /+)}$ were labeled with tamoxifen, rested, subjected to an orotracheal dose of PBS or bleomycin and euthanized 14 days later. Lung cryosections were directly imaged for Rb colors (mCherry, mOrange, Cerulean) with boxed regions shown as close-ups [right; $\mathbf{g}$ ]. In each patch, the number of contiguous labeled cells of the same color was scored and averaged (h; $p=0.0022)$ or binned into groups (i). $n=3$ mice for PBS and $n=4$ for bleomycin groups, three sections per mouse, average of 16 patches per section. One-way ANOVA with Tukey's multiple comparisons test $(\mathbf{c}, \mathbf{d})$ and two-tailed Student's $t$ test $(\mathbf{f}, \mathbf{h})$. Data are averages \pm SD. Source data are provided as a Source Data file. Scale

bars, $25 \mu \mathrm{m}$.

Possible factors contributing to the reduced bleomycin-induced

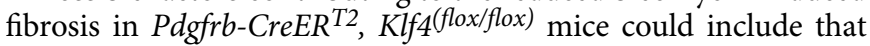
Klf4 deletion in preexisting PDGFR- $\beta^{+}$cells reduces proliferation and/or differentiation into myofibroblasts of this lineage. Thus, Pdgfrb-CreER ${ }^{T 2}, R_{0 S A 26 R^{(Y F P /+)}}$ mice that also carried Klf4 $4^{(f l o x /}$ flox) or were wild type for Klf4 were injected with tamoxifen, rested and then bleomycin was administered. Mice were injected with EdU and $12 \mathrm{~h}$ later, they were euthanized on day 5 or 14 after bleomycin. Histological analysis of lung sections stained for YFP and EdU shows that Klf4 deletion does not significantly alter proliferation of cells of the PDGFR- $\beta^{+}$cell lineage at day 5 (Supplementary Fig. 11b, c) or 14 (Fig. 3f, g). In addition, the percentage of SMA ${ }^{+}$myofibroblasts that derive from PDGFR- $\beta^{+}$ cells is not changed by Klf4 deletion in PDGFR- $\beta^{+}$cells (Fig. 3h, i). Taken together, these findings indicate that, in the context of bleomycin treatment, although Klf4 deletion in PDGFR- $\beta^{+}$cells attenuates lung fibrosis, KLF4 is not required for PDGFR- $\beta^{+}$cell proliferation or differentiation into myofibroblasts.

KLF4 regulates TGF $\beta$ signaling and ECM synthesis in PDGFR$\boldsymbol{\beta}^{+}$cells. To further interrogate mechanisms by which KLF4 in PDGFR- $\beta^{+}$cells may induce lung fibrosis, we conducted studies on isolated lung cells. Pdgfrb-CreER ${ }^{T 2}, \operatorname{ROSA}_{26 R^{(Z s /+)}}$ mice were induced with tamoxifen, and then lung $\mathrm{Zs}^{+}$cells were isolated by FACS and Zs expression was verified in culture (Supplementary Fig. 12). Complementing our findings in mice (Fig. 3b-e), siRNAmediated Klf4 silencing of these isolated cells reduced mRNA and/ or protein levels of key ECM components of the fibrotic response, Colla1, Col3a1 and Fn1 (Fig. 4a-c). TGF $\beta$-mediated SMAD2/3 phosphorylation is the major pathway implicated in organ fibrosis ${ }^{41,42}$, and the PDGF signaling pathway plays a role as well ${ }^{43}$. Klf4 knockdown in isolated lung $\mathrm{Zs}^{+}$cells from $P d g f r b-$ CreER ${ }^{T 2}, \operatorname{ROSA} 26 R^{(Z s /+)}$ mice resulted in downregulation of ligands Tgfbl and Pdgfb and their receptors Tgfbr1, 2 and Pdgfrb, respectively; however, mRNA levels of Pdgfra and Itgb3, known regulators of the ECM and fibrotic response ${ }^{43,44}$, were not altered (Fig. 4a, d). Furthermore, reduction of Klf4 in isolated $\mathrm{Zs}^{+}$cells decreases levels of SMAD3 phosphorylation by $~ 50 \%$ (Fig. 4e, f). To evaluate the in vivo relevance of this finding to fibrotic disease, $P d g f r b-C r e E R^{T 2}, K l f 4^{(f l o x / f l o x)}$ mice were induced with tamoxifen and then exposed to orotracheal bleomycin. Fourteen days later, lung lysates were analyzed by western blot and a similar reduction of phospho-SMAD3 is detected (Fig. 4g, h). Taken together, our data suggest that KLF4 regulates TGF $\beta$-SMAD signaling and thereby ECM synthesis in PDGFR- $\beta^{+}$lung cells in culture and in fibrotic lung disease.
Klf4 deletion in $\mathrm{SMA}^{+}$cells exacerbates pulmonary fibrosis. Given that $\mathrm{SMA}^{+}$cells give rise to $\sim 10 \%$ of bleomycin-induced lung myofibroblasts (Fig. 1b, c) and that KLF4 plays a prominent role in transformation of PDGFR- $\beta^{+}$cells to a profibrotic phenotype (Figs. 3 and 4), we next assessed the role of KLF4 in SMA + cells in fibrogenesis. KLF4 is dynamically upregulated in lung $\mathrm{SMA}^{+}$cells during the early stages of the acute inflammatory phase following bleomycin administration (Supplementary Fig. 13a). To evaluate the effects of deleting Klf4 in airway and vascular SMCs and the very rare myofibroblasts in the basal lung on subsequent development of fibrosis, Acta2-CreER ${ }^{T 2}$, Klf4 (flox/ $f(0 x)$ mice were injected with either tamoxifen or vehicle (corn oil), rested and then treated with a single orotracheal dose of bleomycin. Fourteen days later, mice were euthanized and lungs were processed. Surprisingly, there is a marked increase in lung myofibroblasts with Klf4 deletion in $\mathrm{SMA}^{+}$cells (Fig. 5a). Furthermore, there is an increase in levels of collagen (Fig. 5b, c) and SMA, COL1 and FN1 proteins (Fig. 5d, e). These findings indicate that, in stark contrast to its role in PDGFR- $\beta^{+}$cells (Fig. 3), KLF4 in $\mathrm{SMA}^{+}$cells protects against bleomycin-induced lung fibrosis.

Subsequently, we analyzed the effect of Klf4 deletion in preexisting $\mathrm{SMA}^{+}$cells on proliferation and fate of this lineage and on myofibroblast proliferation following bleomycin treatment. Acta2-CreER $R^{T 2}, R_{0 S A 26 R^{(Z s /+)}}$ mice also carrying Klff(flox $/$ $f(l o x)$ or wild type for Klf4 were induced with tamoxifen, rested, treated with orotracheal bleomycin and then euthanized 14 days later. Twelve hours prior to euthanasia, EdU was administered. Lungs were harvested and stained for SMA, EdU and/or nuclei (DAPI) and directly imaged for Zs. The percentage of $\mathrm{Zs}^{+}$cells that are proliferative $\left(\mathrm{EdU}^{+}\right.$; Supplementary Fig. 13b, c) and the percentage of myofibroblasts that derive from $\mathrm{Zs}^{+}$cells (Fig. 5f, g) are not altered by Klf4 deletion in preexisting $\mathrm{SMA}^{+}$cells as previously observed with Klf4 deletion in preexisting PDGFR- $\beta^{+}$ cells (Fig. $3 \mathrm{f}-\mathrm{i}$ ). Interestingly, the percentage of total myofibroblasts that are proliferative is substantially increased in tamoxifen-induced Acta2-CreER ${ }^{T 2}$, Klf4(flox/flox) mice (Fig. 5h, i). In sum, these observations indicate that KLF4 in $\mathrm{SMA}^{+}$cells does not cell autonomously regulate proliferation or differentiation to myofibroblasts during bleomycin-induced lung injury, but deletion of Klf4 in $\mathrm{SMA}^{+}$cells does exacerbate myofibroblast proliferation and accumulation and fibrosis.

Reduction of KLF4 in SMA ${ }^{+}$cells induces CCL2 expression. To further investigate mechanisms underlying the protective role of 

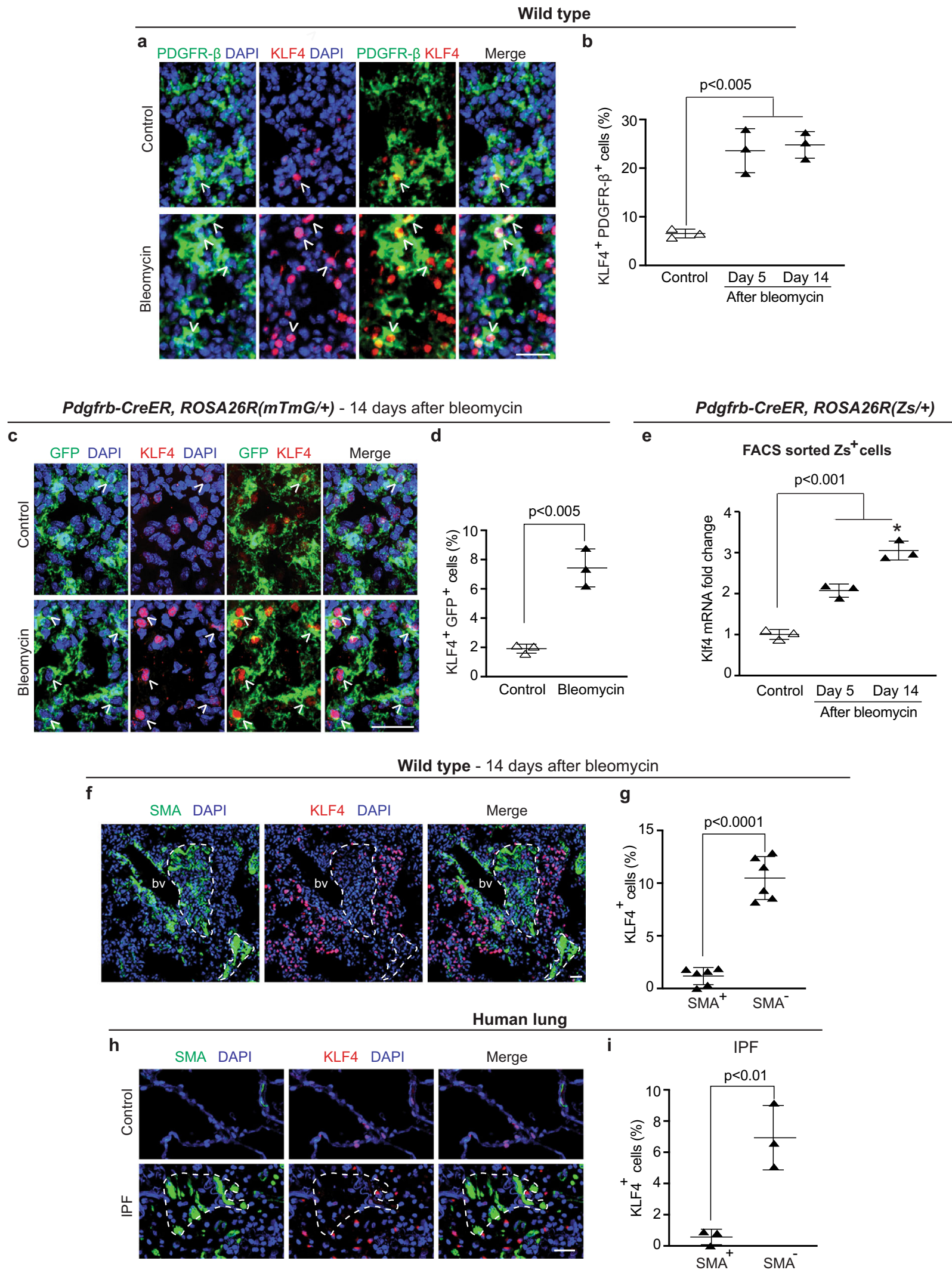

KLF4 in SMA ${ }^{+}$cells during lung fibrosis, we evaluated the effects of siRNA-mediated Klf4 knockdown in murine airway SMCs. In contrast to the results with PDGFR- $\beta^{+}$cells (Fig. 4), Klf4 silencing in SMCs does not alter levels of ECM components Colla1, Col3al or Fn1 (Fig. 6a) suggesting that Klf4 deletion in SMCs may not directly influence ECM deposition by SMCs during fibrosis. Thus, we explored potential noncell autonomous effects, starting with the expression of select growth factors, cytokines, chemokines previously implicated in fibrosis 45,46 . Transcript levels of Tgfb1, Pdgfb, Tnfa, interleukins $1 b, 5$ and 6 and the relevant TF Nfkb do not change with Klf4 knockdown in SMCs (Fig. 6b), whereas mRNA and secreted protein levels of CCL2 
Fig. 2 KIf4 is upregulated in PDGFR- ${ }^{+}$cells after bleomycin treatment. $\mathbf{a}$, b, Wild-type mice were or were not subjected to an orotracheal dose of bleomycin, and 5 or 14 days thereafter, lung cryosections were stained for PDGFR- $\beta$ and KLF4. Images at 14 days (a) were used to quantify the percent of PDGFR- $\beta^{+}$lung cells expressing KLF4 (b). For each treatment group, $n=3$ mice, three sections per mouse and an average of 53 PDGFR- $\beta^{+}$cells per section. Day $5(p=0.0013)$, day $14(p=0.0009)$ vs. control. c, d Pdgfrb-CreER ${ }^{T 2}, R O S A 26 R^{(m T m G /+)}$ mice were induced with tamoxifen, rested and given an orotracheal dose of PBS or bleomycin. Fourteen days later, lung cryosections were stained for GFP (fate marker) and KLF4 (c), and the percent of $\mathrm{GFP}^{+}$cells that are KLF4+ ${ }^{+}$was quantified (d). For each treatment group, $n=3$ mice, three sections per mouse and an average of $40 \mathrm{GFP}+$ cells per section. $p=0.002$. e Pdgfrb-CreER ${ }^{T 2}, R O S A 26 R^{(Z s /+)}$ mice were induced with tamoxifen, rested, treated or not treated with orotracheal bleomycin, and 5 or 14 days later, $\mathrm{Zs}^{+}$cells were isolated by FACS and subjected to qRT-PCR for Klf4 and Gapdh. Klf4 mRNA relative to Gapdh and normalized to untreated control is shown. For each treatment group, $n=3$ mice with qRT-PCR done in triplicate. Day $5(p=0.0007)$, day $14(p<0.0001)$ vs. control; * vs. day $5, p=0.012$. $\mathbf{f - i}$ Lung stains for SMA and KLF4 of cryosections at 14 days after bleomycin-induction of wild-type mice (f) and of paraffin sections from human control and IPF patients (h). bv, blood vessel. Fibrotic areas demarked by dashed lines have minimal KLF4 staining. In $\mathbf{g}$ and $\mathbf{i}_{\text {, the percent of SMA }}^{+}$and SMA parenchymal cells that are KLF4 ${ }^{+}$is quantified from sections as in $\mathbf{f}$ and $\mathbf{h} ; n=6$ mice, three sections per mouse, average of $178 \mathrm{SMA}^{+}$cells and $910 \mathrm{SMA}$ cells per section, $p<0.0001$ and $n=3$ humans, three sections per human, average of $110 \mathrm{SMA}^{+}$cells and $729 \mathrm{SMA}^{-}$cells per section, $p=0.0066$. Oneway ANOVA with Tukey's multiple comparisons test $(\mathbf{b}, \mathbf{e})$ and two-tailed Student's $t$ test $(\mathbf{d}, \mathbf{g}, \mathbf{i})$. Data are averages \pm SD. Source data are provided as a Source Data file. Scale bars, $25 \mu \mathrm{m}$.

(also known as monocyte chemoattractant protein-1) are markedly increased (Fig. 6c, d). In notable contrast, Klf4 knockdown in PDGFR- $\beta^{+}$cells does not alter Ccl 2 expression (Supplementary Fig. 14a). CCL2 is a chemokine for myeloid cells, and treatment with anti-CCL2 antiserum or gene transfer of a dominant negative $\mathrm{Ccl} 2$ attenuates lung fibrosis in mice ${ }^{47-49}$. As a validation of the in vivo relevance of our findings showing KLF4 regulation of $\mathrm{Ccl} 2$ in cultured SMCs, deletion of Klf4 in $\mathrm{SMA}^{+}$cells increases both CCL2 in lung lysates and the number of lung cells expressing the macrophage marker CD68 in bleomycin-treated mice (Fig. 6e-h). Together, these findings suggest Klf4 deletion in $\mathrm{SMA}^{+}$cells worsens bleomycin-induced lung fibrosis at least partly by increasing secretion of CCL2.

Klf4 silenced SMCs induce fibrotic changes in PDGFR- $\beta^{+}$cells via CCR2. In addition to promoting macrophage accumulation, we queried whether conditioned medium from siKLF4-treated SMCs induces profibrotic transformation of PDGFR- $\beta^{+}$lung cells via the CCL2 receptor CCR2. To this end, $\mathrm{Zs}^{+}$cells were isolated by FACS from lungs of tamoxifen-induced Pdgfrb-CreER ${ }^{T 2}$, ROSA26R ${ }^{(\mathrm{Zs} /+)}$ mice. These $\mathrm{Zs}^{+}$cells were cultured in conditioned medium from siKlf4-treated SMCs (experimental medium) or from scrambled (Scr) siRNA-treated SMCs (control medium) for $24 \mathrm{~h}$. $\mathrm{Zs}^{+}$cells cultured in experimental medium are $\sim 2.5$ more proliferative as determined by EdU incorporation (Fig. 6i, j). Moreover, experimental medium induces these cells to express increased levels of Klf4, Colla1, Col3a1 and Tgfb1 (Fig. 6k), consistent with previous findings that KLF4 mediates expression of collagens and Tgfb1 in PDGFR- $\beta^{+}$cells (Figs. 3b-e and $4 \mathrm{a}-\mathrm{c}$ ). Furthermore, Ccr2 transcript levels are elevated by $\sim 12$-fold in $\mathrm{Zs}^{+}$cells cultured in experimental as opposed to control medium (Fig. 6k), and thus, we evaluated whether collagen levels are regulated by the CCL2-CCR2 axis in these cells. $\mathrm{Zs}^{+}$cells were exposed to Scr RNA or siRNA against Ccr2 and then incubated in experimental conditioned medium. Ccr2 knockdown in $\mathrm{Zs}^{+}$cells downregulates Colla1 and Col3a1 transcripts by more than 90 -fold with culturing in experimental conditioned medium (Fig. 6l) but has no effect with culturing in standard medium (low glucose DMEM with supplements, see "Methods"; Supplementary Fig. 14b). Collectively, these data suggest that CCL2 secreted by siKlf4-treated SMCs signal through the CCR2 receptor on PDGFR- $\beta^{+}$cells to induce collagen expression.

KLF4 differentially regulates $C c l 2$ and $T g f b 1$ in SMCs and PDGFR- $\boldsymbol{\beta}^{+}$cells. To gain further mechanistic insights into KLF4mediated differential regulation of downstream targets in SMCs and PDGFR- $\beta^{+}$cells, these cells were treated with Scr RNA or siRNA targeting Klf4 and then subjected to bulk RNA-seq (Supplementary Data 1 and 2). Consistent with qRT-PCR and western/dot blot analyses (see Fig. 6b-f and Supplementary Fig. 14a), Ccl2 levels were increased $(\sim 5.7$-fold, padj $<0.005)$ with knockdown of Klf4 in SMCs whereas in PDGFR- $\beta^{+}$cells, siKlf4 treatment did not alter Ccl2 levels. We next sought TFs that bind $\mathrm{Ccl} 2$ cis-regulatory regions, induce expression and are differentially expressed with Klf4 knockdown, in a similar manner to Ccl2. To this end, using the TRANSFAC database, 407 TFs were identified that are predicted to bind between $-5000 \mathrm{bp}$ to $+100 \mathrm{bp}$ (transcription start site of $\mathrm{Ccl} 2$ is at position +1 ) (Supplementary Data 3). Using ingenuity pathway analysis, this data set was aligned to the RNA-seq data, yielding expression data for 234 and 230 TFs in SMCs and PDGFR- $\beta^{+}$cells, respectively, that are predicted to bind $\mathrm{Ccl} 2$, and Volcano plots were generated (Fig. 7a, b). Of the five most highly upregulated transcripts in SMCs, three of these genes, ETS variant TF 4 (Etv4), Foxm1 and MYB proto-oncogene like 2 (Mybl2), are not significantly upregulated in PDGFR- $\beta^{+}$cells, but there is a trend to an increased Etv4 level in PDGFR- $\beta^{+}$cells (Fig. 7c).

We elected to further investigate Foxm1 based on these findings and recent studies demonstrating that KLF4 negatively regulates Foxm1 expression in gastrointestinal cancer cell lines, FOXM1 positively regulates $\mathrm{Ccl} 2$ expression in hepatocytes and Foxm 1 deletion in COL1a2 ${ }^{+}$cells attenuates bleomycin-induced lung fibrosis ${ }^{50-52}$. As with RNA-seq, using qRT-PCR, we found that siRNA targeting Klf4 increases Foxm1 transcript levels in SMCs but not in PDGFR- $\beta^{+}$cells (Fig. $7 \mathrm{~d}$ ). FOXM1 protein is increased in SMCs by $5.0 \pm 0.4$-fold with siKlf4 treatment and in lung lysates by $5.4 \pm 0.8$-fold with tamoxifen-treatment of Acta2CreER, Klf4(flox/flox) mice (Fig. 7e-h). Importantly, concomitant knockdown of Klf4 and Foxm1 in SMCs prevents the robust upregulation of $\mathrm{Ccl} 2$ observed with only Klf4 silencing (Fig. 7i). Chromatin immunoprecipitation (ChIP) assay with an anti-KLF4 or isotype control antibody suggests that KLF4 robustly binds the promoter region of Foxm1 (Fig. 7j). In addition, we found that pyruvate dehydrogenase kinase 1 , which is implicated as a positive regulator of Foxm 1 mRNA levels in a human lung fibroblast cell line $\mathrm{e}^{51}$, is upregulated in SMCs but not PDGFR- $\beta^{+}$ cells with Klf4 knockdown (Supplementary Fig. 15).

As with the Foxm1-Ccl2 axis, upon Klf4 knockdown, Tgfb1 levels are differentially altered in lung PDGFR- $\beta^{+}$cells (downregulated; Fig. 4d) and SMCs (unchanged; Fig. 6b). It was previously reported that in vimentin ${ }^{+}$noncardiomyocyte cells isolated from the neonatal mouse heart, KLF4 binds the Tgfb1 promoter and induces transcription ${ }^{29}$. We performed the ChIP assay using an anti-KLF4 or isotype control antibody and found 
Pdgfrb-CreER, KIf4(flox/flox) - 14 days after bleomycin

a
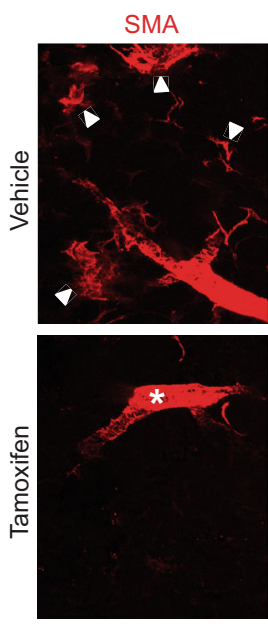

d

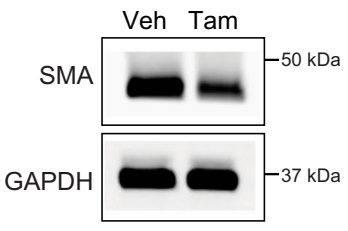

b
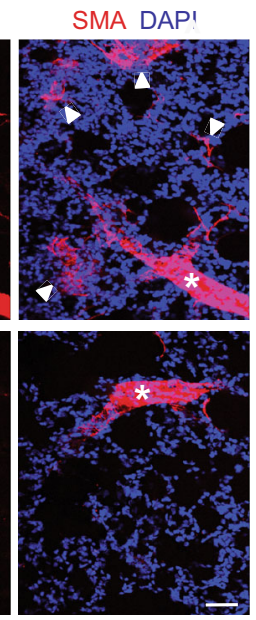

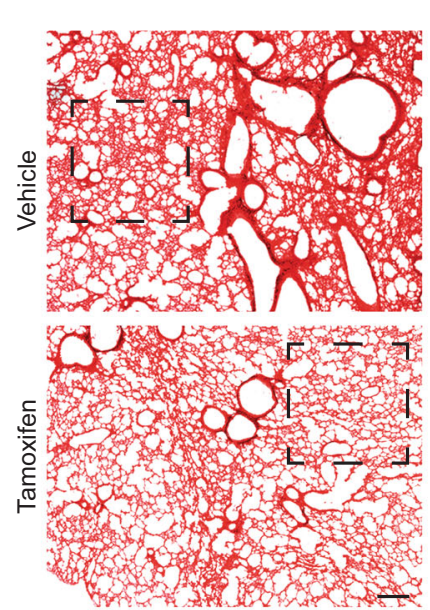

Picrosirius red stain

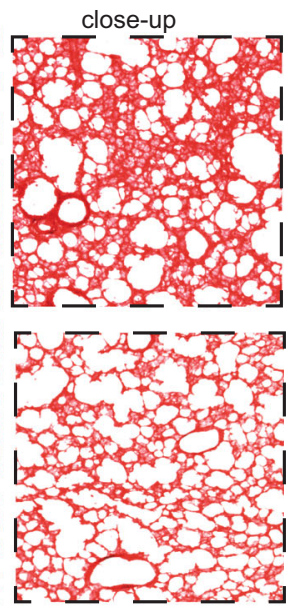

C

Sircol assay

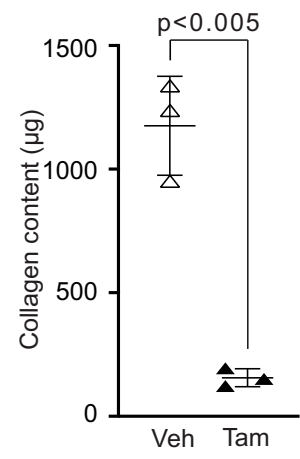

Pdgfrb-CreER, ROSA26R(YFP/+) - 14 days after bleomycin
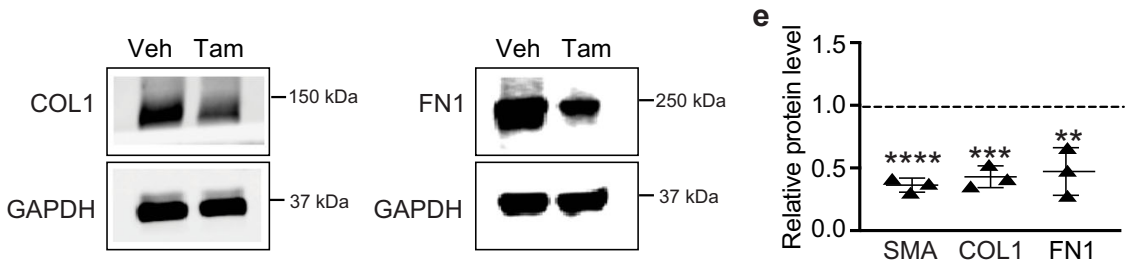

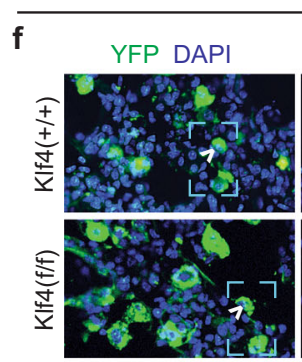

h

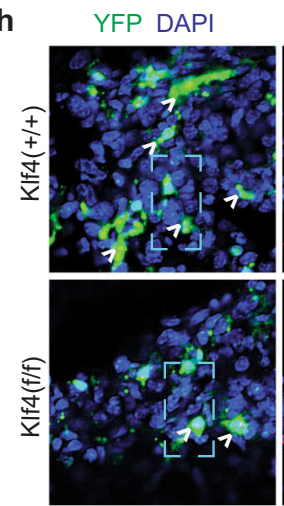

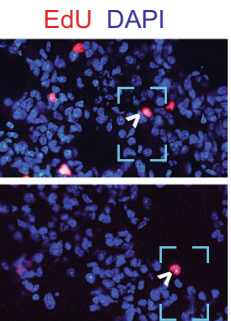

SMA DAPI
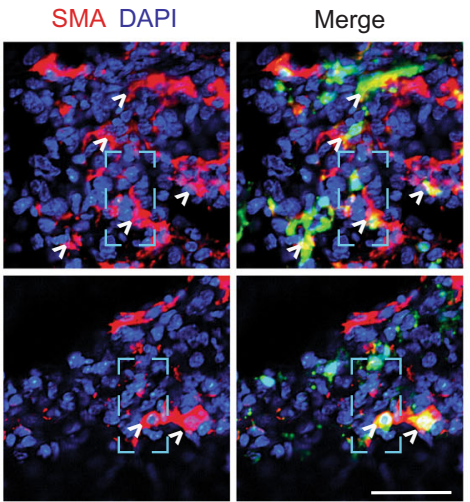

that KLF4 binding to the $T g f b 1$ promoter region was increased in PDGFR- $\beta^{+}$cells $(5.7 \pm 0.7$-fold) but not in SMCs (Fig. 7k). Next, we queried whether this differential KLF4 binding is due to differences in chromatin accessibility which would facilitate interactions with TFs. Indeed, ChIP of the Tgfb1 promoter with an antibody targeting acetylated histone H3 lysine9 (H3K9Ac), which marks open chromatin, demonstrates $50 \%$ higher signal in PDGFR- $\beta^{+}$cells than SMCs (Fig. 7l). Taken together, our data indicate that KLF4 differentially regulates profibrotic mediators in distinct lung cell types.

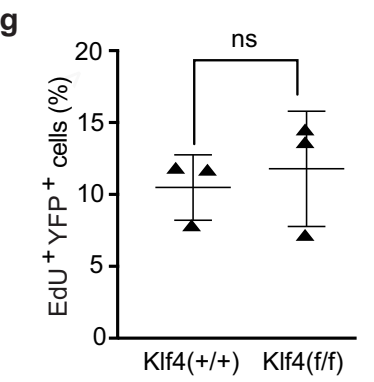

i
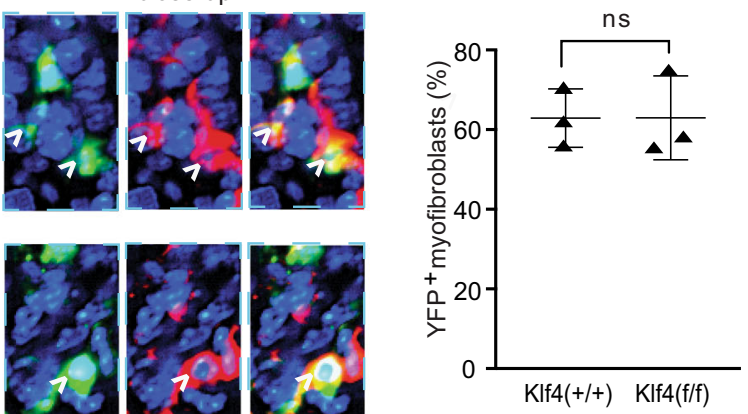

\section{Discussion}

In the healthy lung, epithelial cells lining the airways and alveoli are a critical line of defense against toxins. Chronic insults to these cells can trigger the fibrotic response largely via deleterious effects on underlying mesenchymal cells. During the pathogenesis of lung fibrosis, mesenchymal cells are implicated as the major source of excessive myofibroblasts and ECM components. Although aberrant signaling from epithelium to mesenchyme is considered a key element of the pathogenesis of lung fibrosis, the role of signaling between different mesenchymal cell types is not well delineated. Herein, using 
Fig. 3 Klf4 deletion in PDGFR- ${ }^{+}$cells attenuates bleomycin-induced myofibroblast accumulation and lung fibrosis. a-e $P d g f r b-C r e E R^{T 2}, K I f 4(f l o x / f l o x)$ mice were induced with either vehicle (Veh; corn oil) or tamoxifen (Tam), rested and treated with bleomycin. Lungs were analyzed 14 days later. Cryosections were stained for SMA and nuclei (DAPI) in (a) and with Picrosirius red (b) with dashed boxes shown as close-ups on the right. Closed arrowheads and asterisks indicate SMA+ fibrotic patches and blood vessels, respectively. $n=3$ mice for vehicle and $n=6$ mice for tamoxifen inductions. Collagen content of the right lung was measured using the Sircol assay (c); $n=3$ mice in duplicate, $p=0.001$. Western blot analysis of lung lysates for

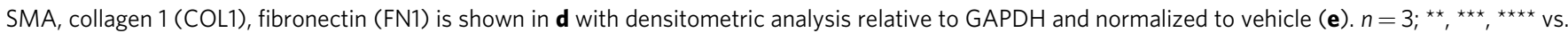
vehicle, $p=0.0086, p=0.0003, p<0.0001$, respectively. f-i Pdgfrb-CreER ${ }^{T 2}, R O S A 26 R^{(Y F P /+)}$ mice carrying KIf4(flox/flox) or wild type for KIf4 were induced with tamoxifen, rested, treated with bleomycin and lungs were analyzed 14 days later. Mice were injected with EdU $12 \mathrm{~h}$ prior to euthanasia. Lung cryosections were stained for YFP (fate marker) and nuclei (DAPI) as well as for EdU (f) or SMA (h) with boxed regions shown as close-ups on the right. Open arrowheads indicate $\mathrm{YFP}^{+}$cells that are also $\mathrm{EdU}^{+}(\mathbf{f})$ or $\mathrm{SMA}^{+}(\mathbf{h})$. The percentage of $\mathrm{YFP}^{+}$cells that are $\mathrm{EdU}^{+}$or are $\mathrm{SMA}^{+}$myofibroblasts was quantified in $\mathbf{g}$ and $\mathbf{i}$, respectively. ns not significant. For each genotype, we analyzed $n=3$ mice, three sections per mouse, and on average 41 YFP + cells (g) or 68 myofibroblasts (i) per section. Two-tailed Student's $t$ test was used for all analysis. Data are averages \pm SD. Source data are provided as a Source Data file. Scale bars, $200 \mu \mathrm{m}(\mathbf{b}), 50 \mu \mathrm{m}(\mathbf{a}), 25 \mu \mathrm{m}(\mathbf{f}, \mathbf{h})$.
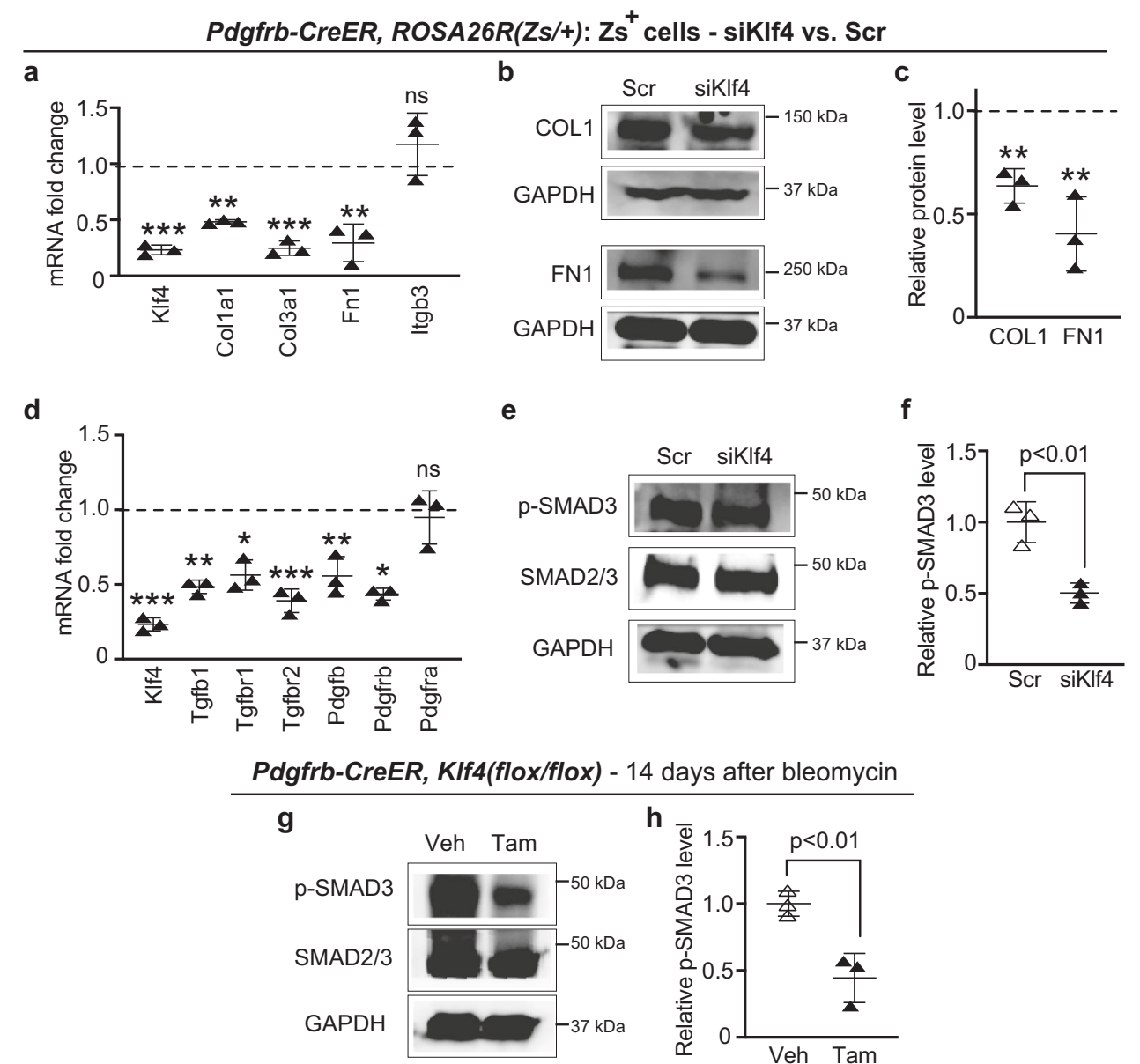

Fig. 4 KIf4 reduction in PDGFR- ${ }^{+}$cells attenuates levels of TGF $\beta$ pathway members and signaling and ECM components. a-f $Z s^{+}$cells were isolated from the lungs of tamoxifen-induced Pdgfrb-CreERT2, ROSA26R(Zs/+) mice and then subjected to siRNA-mediated knockdown with siKlf4 vs. scrambled (Scr). In a and $\mathbf{d}$, levels of indicated transcripts relative to Gapdh were determined by qRT-PCR and normalized to Scr. $n=3$ in triplicate. *, Tgfbr1 $(p=0.017)$, Pdgfrb $(p=0.010) ;{ }^{\star \star}, \operatorname{Col1a1}(p=0.0035), \operatorname{Fn} 1(p=0.0042), \operatorname{Tgfb} 1(p=0.0034), \operatorname{Pdgfb}(p=0.0045) ;{ }^{\star \star \star}$, Klf4 $(p=0.0006)$, Col3a1 ( $p=0.0004)$, Tgfbr2 ( $p=0.0007$ ); ns (not significant) vs. Scr. In $\mathbf{b}$ and $\mathbf{e}$ and $\mathbf{c}$ and $\mathbf{f}$, western blots for indicated proteins and corresponding densitometry relative to GAPDH and normalized to Scr are shown, respectively. $n=3 .{ }^{\star \star}$, COL1 $(p=0.0016)$, FN1 $(p=0.0045)$ vs. Scr in $\mathbf{b}$ and $p=0.0057$ in

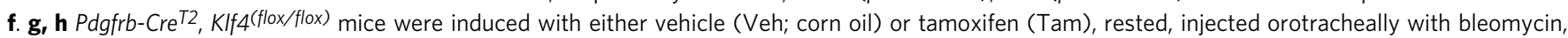
and 14 days later, lungs were harvested. Western blots of whole lung lysates for phosphorylated (p)-SMAD3, total SMAD2/3 and GAPDH are shown in $\mathbf{g}$ with densitometries of $p$-SMAD3 relative to SMAD2/3 and normalized to Veh (h). $n=3, p=0.0095$. Two-tailed Student's $t$ test was used for all analysis. Data are averages \pm SD. Source data are provided as a Source Data file.

bleomycin-induced lung fibrosis, we demonstrate that PDGFR- $\beta^{+}$ cells are the major source of myofibroblasts (Fig. 1), and KLF4 in PDGFR- $\beta^{+}$-derived cells is critical for the accumulation of excess myofibroblasts and ECM (Figs. 2-4). In stark contrast, KLF4 in $\mathrm{SMA}^{+}$cells is protective, and reduction of Klf4 in these cells enhances the number of lung myofibroblasts and noncell autonomously induces PDGFR- $\beta^{+}$cells to generate excess ECM components (Figs. 5 and 6). Mechanisms underlying cell-type-specific effects of KLF4 in SMCs and PDGFR- $\beta^{+}$cells are evaluated (Fig. 7) and summarized in Fig. $7 \mathrm{~m}$. 


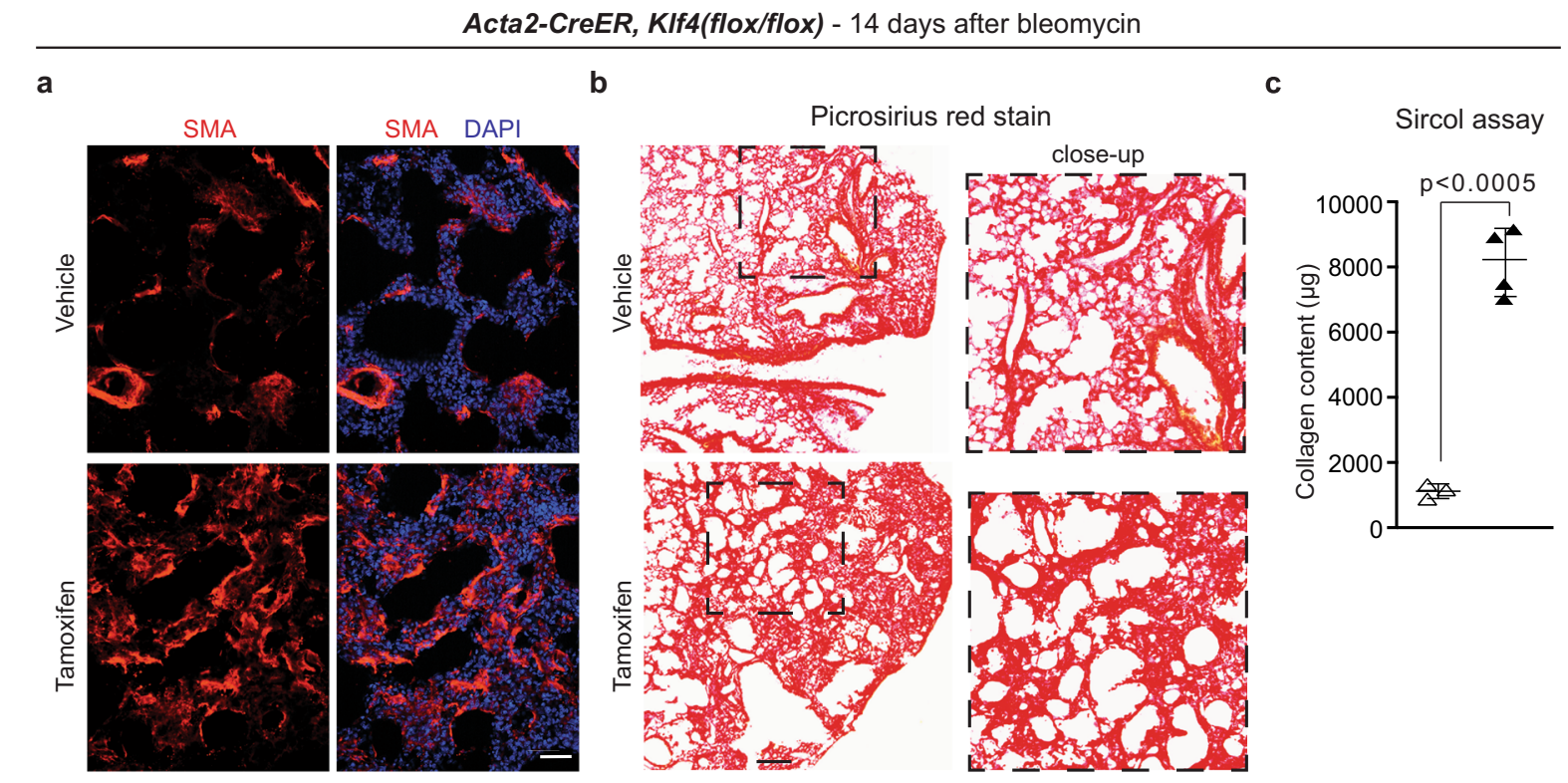

d

e
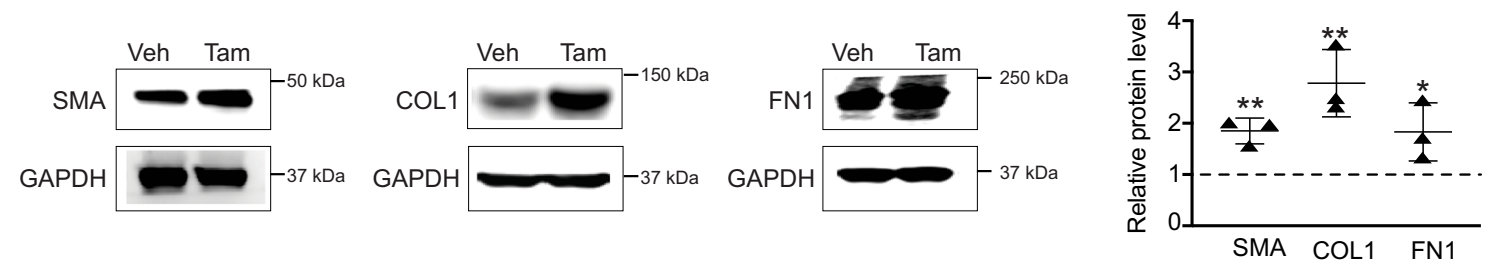

Acta2-CreER, ROSA26R(Zs/+) - 14 days after bleomycin

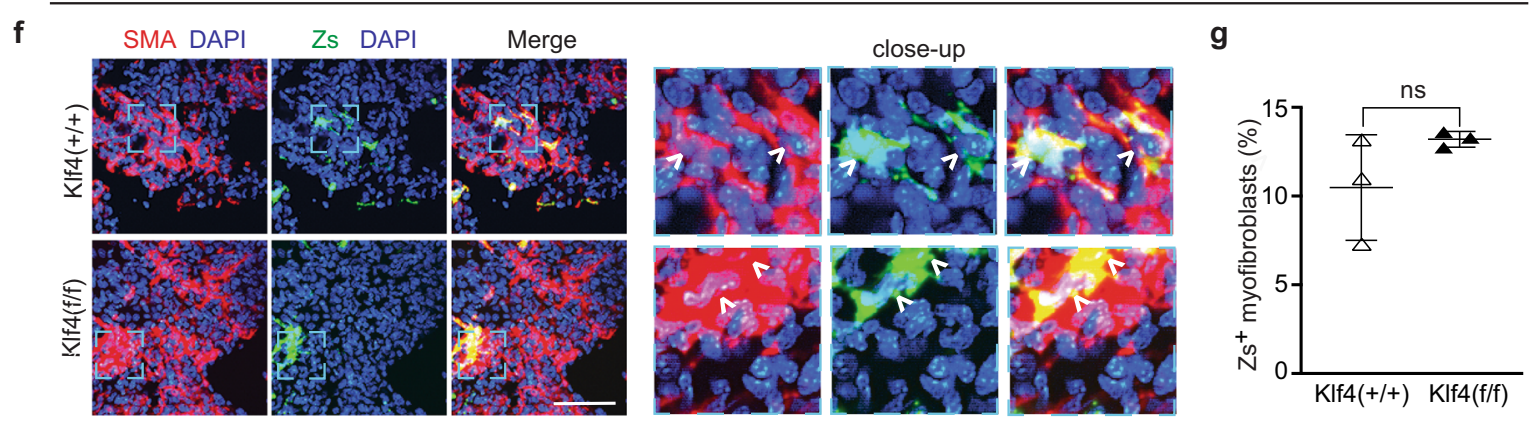

Acta2-CreER - 14 days after bleomycin

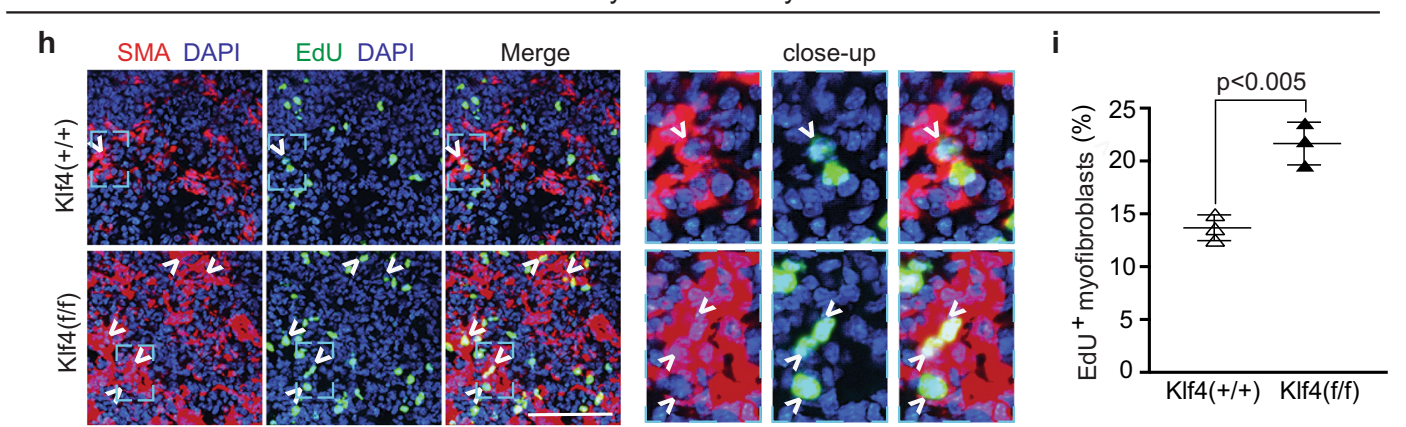

Myofibroblasts are classically considered a key cell type in the pathobiology of lung fibrosis-important for lung contraction and accumulation of excess ECM-but their cellular origins are incompletely defined. Fate mapping studies suggest that diverse mesenchymal cell types give rise to a substantial portion of pathological myofibroblasts whereas it is controversial whether epithelial cells contribute ${ }^{13-17,41,53}$. These source mesenchymal cell populations include $\mathrm{ADRP}^{+}$lipofibroblasts, FoxD1 ${ }^{+}$ progenitor-derived pericytes, Gli1 ${ }^{+}$MSC-like cells and Axin2 ${ }^{+}$ myofibrogenic progenitors and the latter three cell populationspericytes, MSC-like cells and Axin2 ${ }^{+}$progenitors-express PDGFR- $\beta$. Following a single bleomycin dose, fate mapping of $\mathrm{Gli1}^{+}$cells suggests that Gli1 ${ }^{+}$PDGFR- $\beta^{+}$MSC-like cells give rise to $37 \%$ of lung myofibroblasts at 14 day $^{15}$ whereas we find that 


\begin{abstract}
Fig. 5 KIf4 deletion in SMA ${ }^{+}$cells promotes myofibroblast accumulation and exacerbates bleomycin-induced lung fibrosis. a-e $A c t a 2-C r e E R{ }^{T 2}, K I f 4(f l o x /$ flox) mice were induced with either vehicle (Veh; corn oil) or tamoxifen (Tam), rested and treated with bleomycin. Lungs were analyzed 14 days later. Cryosections were stained for SMA and nuclei (DAPI) in $\mathbf{a}$ and with Picrosirius red in $\mathbf{b}$ with dashed boxes shown as close-ups on the right. $n=3$ mice. Collagen content of the right lung was measured using the Sircol assay (c); $n=3$ mice for vehicle and $n=4$ mice for tamoxifen inductions in duplicate. $p=0.0001$. Western blot analysis of lung lysates for SMA, COL1 and FN1 is shown in $\mathbf{d}$ with densitometric analysis relative to GAPDH and normalized to vehicle $(\mathbf{e}) . n=3{ }^{\star}{ }^{*} \mathrm{FN} 1(p=0.044) ;{ }^{\star \star} \mathrm{SMA}(p=0.0042)$, COL1 (0.0093) vs. vehicle. $\mathbf{f}-\mathbf{i}$ Acta2-CreER ${ }^{T 2}$ mice carrying KIf4(flox/flox) or wild type for KIf4 were induced with tamoxifen, rested and treated with bleomycin, and lungs were analyzed 14 days later. In $\mathbf{f}$, mice were also $R O S A 26 R^{(Z s /+)}$, and in $\mathbf{h}$, mice were injected with EdU $12 \mathrm{~h}$ prior to euthanasia. Lung cryosections were stained for SMA and nuclei (DAPI) and either directly imaged for Zs ${ }^{+}(\mathbf{f})$ or also stained for EdU (h) with boxed regions shown as close-ups on the right. Arrowheads indicate SMA+ myofibroblasts that are also Zs (f) or EdU $+(\mathbf{h})$. The percentage of myofibroblasts that are $\mathrm{Zs}^{+}$or $\mathrm{EdU}^{+}$was quantified in $\mathbf{g}$ and $\mathbf{i}$, respectively. $n=3$ mice, four sections per mouse, and on average $132 \mathrm{SMA}+$ myofibroblasts per section. ns, not significant $(\mathbf{g})$ and $p=0.0042$ (i). Two-sided Student's $t$ test was used for all analysis. Data are averages \pm SD. Source data are provided as a Source Data file. Scale bars, $200 \mu \mathrm{m}$ (b), $50 \mu \mathrm{m}(\mathbf{a}), 25 \mu \mathrm{m}(\mathbf{f}, \mathbf{h})$.
\end{abstract}

PDGFR- $\beta^{+}$cells give rise to $\sim 85 \%$ of myofibroblasts at day 7 and $\sim 70 \%$ of myofibroblasts at day 14 (Fig. 1). Thus, both Gli1 ${ }^{+}$PDGFR- $\beta^{+}$and Gli1-PDGFR- $\beta^{+}$cell populations are likely important sources of pathological myofibroblasts. Albeit to a considerably lesser extent than PDGFR- $\beta^{+}$cells, recent studies suggest that PDGFR- $\alpha^{+}$cells contribute ${ }^{14,54}$. One of these studies, by Zepp et al., also implicates peribronchiolar Axin $2^{+}$PDGFR- $\alpha^{-}$ PDGFR- $\beta^{+}$cells that have enriched RNA levels of Acta2, transgelin and desmin as an important source of bleomycin-induced myofibroblasts ${ }^{14}$. Interestingly, immunohistochemistry reveals that less than $10 \%$ of Axin $2+$ cells surrounding airways or blood vessels express SMA or SM22 $\alpha$, and thus, the authors suggest that the Axin ${ }^{+}$myofibrogenic cells may be primed for, but are not committed to, SMC or myofibroblast lineages ${ }^{14}$. In light of these prior findings, it is notable that a study by El Agha et al. suggests that $\mathrm{SMA}^{+}$cells are not a source of bleomycin-induced lung myofibroblasts ${ }^{13}$, and our fate mapping results indicate that only $\sim 10 \%$ of lung myofibroblasts derive from preexisting $\mathrm{SMA}^{+}$cells (Fig. 1). Thus, Acta2-CreER may not efficiently mark the Axin2 ${ }^{+}$ myofibrogenic progenitor cell population and/or Axin2 ${ }^{+}$Acta2cells may be the primary Axin $2^{+}$myofibrogenic progenitors.

The potential significance of the $\sim 15 \%$ decrease in PDGFR- $\beta$ derived myofibroblasts during the second week after bleomycin exposure in our studies is not delineated nor is the underlying mechanism. Possible mechanisms include an increased contribution of another source during this time period or apoptosis or SMA downregulation in the PDGFR- $\beta^{+}$cell lineage. Our multicolor clonal analysis suggests the large contribution of PDGFR- $\beta^{+}$cells to the pathological myofibroblast pool is through clonal expansion of PDGFR- $\beta^{+}$cells with limited mixing of clones. This result is similar to what has been shown with the lineage deriving from cells that express the mesenchyme marker TBX4 $4^{55}$

Mesenchymal cells change profoundly during the pathogenesis of lung fibrosis, and our results indicate that KLF4, a key regulator of cell transitions, is profibrotic in PDGFR- $\beta^{+}$cells and, through noncell autonomous effects, is antifibrotic in $\mathrm{SMA}^{+}$cells (Figs. 3 and 5). Such cell-type-specific effects of KLF4 likely underlie a large part of the disparate effects of KLF4 in renal fibrosis $^{30}$. Interestingly, in the carotid artery adventitia, deletion of $\mathrm{Klf} 4$ in SMC-derived SCA1 ${ }^{+}$SMC marker- progenitors results in elevated collagen levels and expansion of this vascular layer ${ }^{56}$. In the lung, KLF4 is implicated in the normal perinatal accumulation of myofibroblasts as $K l f 4^{(-/-)}$neonates lack alveolar myofibroblasts, and expression of KLF4 in lung fibroblasts isolated from Klf4 null mice induces expression of SMA, collagen and fibronectin ${ }^{23}$. In contrast, KLF4 overexpression either in vivo (transgenic mice) in unspecified cell types reduces bleomycininduced lung fibrosis or in cultured human alveolar epithelial cells attenuates TGF $\beta 1$-induced downregulation of the epithelial marker E-cadherin and upregulation of fibronectin ${ }^{33}$.
Our studies indicate that upon bleomycin treatment, KLF4 expression is enhanced in PDGFR- $\beta^{+}$cells and regions of the lung adjacent to $\mathrm{SMA}^{+}$fibrotic patches, but in agreement with a recent paper $^{33}$, this expression is markedly reduced within the $\mathrm{SMA}^{+}$fibrotic patches of the human IPF or murine lung (Fig. 2). These findings suggest that KLF4 may be important in the initial phases of fibrosis but superfluous once fibrotic patches are established. Klf4 deletion in PDGFR- $\beta^{+}$cells attenuates bleomycin-induced accumulation of myofibroblasts and lung fibrosis (Fig. 3). In a distinct model of lung injury, we recently found that Klf4 deletion in PDGFR- $\beta^{+}$cells prevents hypoxiainduced expansion of vascular SMCs and pulmonary hypertension $^{57}$, and herein, similar to our results with bleomycin, we observed that deletion of Klf4 in PDGFR- $\beta^{+}$cells markedly reduces hypoxia-induced lung myofibroblasts (Supplementary Fig. 7). Surprisingly, with bleomycin treatment, we did not detect an effect of Klf4 deletion in PDGFR- $\beta^{+}$cells on proliferation and percent contribution to $\mathrm{SMA}^{+}$myofibroblasts even though there is an overall reduction in myofibroblasts and SMA protein levels. Bleomycin treatment of mice stimulates lung PDGFR- $\beta^{+}$cells to express collagen, and Klf4 knockdown in PDGFR- $\beta^{+}$cells reduces ECM component levels (Figs. 1 and 4 ). Thus, we suggest that in PDGFR- $\beta^{+}$cells, bleomycin-induced KLF4 cell autonomously enhances ECM production and speculate that the resulting increased stiffness of the lung parenchyma contributes to the conversion of select populations of both PDGFR- $\beta^{+}$and PDGFR$\beta^{-}$cells to $\mathrm{SMA}^{+}$myofibroblasts. This hypothesis is consistent with the recent finding that human placental pericytes upregulate SMA expression with culturing on stiff polyacrylamide hydrogels 58

The TGF $\beta$ pathway is widely implicated in the pathogenesis of tissue fibrosis ${ }^{41}$ - even referred to as the master regulator of fibrosis by some investigators ${ }^{42}$-and our findings indicate that KLF4 induces TGF $\beta$ signaling during this process in the lung. We previously demonstrated a key role of the TGF $\beta$ pathway in PDGFR- $\beta^{+}$pericytes for embryonic brain morphogenesis ${ }^{59}$, and herein, Klf4 deletion in PDGFR- $\beta^{+}$cells prior to bleomycin administration is shown to downregulate phosphorylation of the TGF $\beta$ effector SMAD3 in the lung (Fig. 4). In addition, Klf4 knockdown in isolated PDGFR- $\beta^{+}$cells reduces levels of TGF $\beta$ pathway members-including the ligand Tgfb1, receptors Tgfbr1/ $\mathrm{r} 2$ and phospho-SMAD3 - and collagens 1a1, 3a1 and fibronectin (Fig. 4). In complementary experiments with vimentin ${ }^{+}$noncardiomyocyte cells of the neonatal mouse heart, KLF4 has been shown to activate the TGF $\beta 1$ promoter and enhance both SMA levels and angiotensin II-induced expression of Colla1 and Col3a $1^{29}$. In contrast, a prior study of cells isolated from the rodent lung by enzymatic digestion and cultured under conditions resulting in fibroblast-like morphology reported that KLF4 binds SMAD3 and thereby, inhibits activation of the Acta2 promoter $^{31,32}$. Importantly, latent TGF $\beta$ is activated by many 
SMCs - siKIf4 vs. Scr

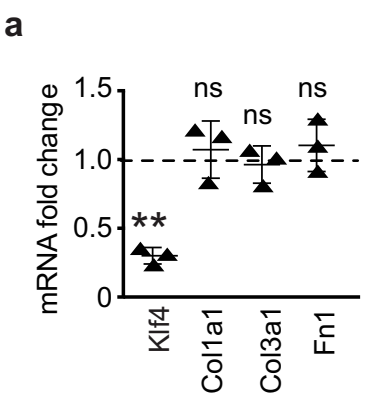

b

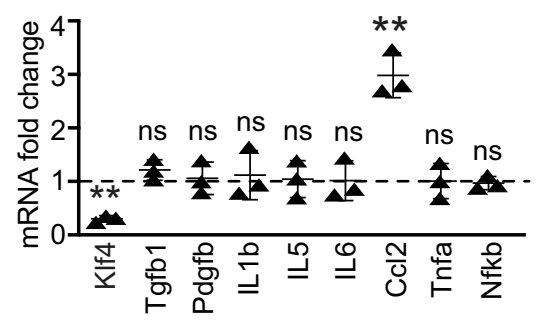

SMC conditioned medium (CM)

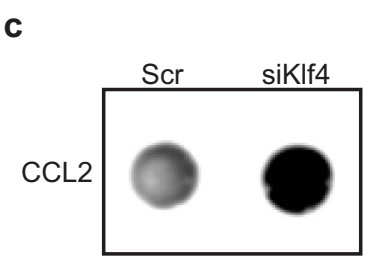

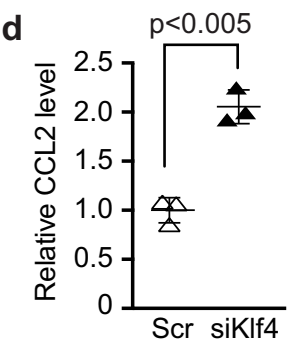

Acta2-CreER - 14 days after bleomycin

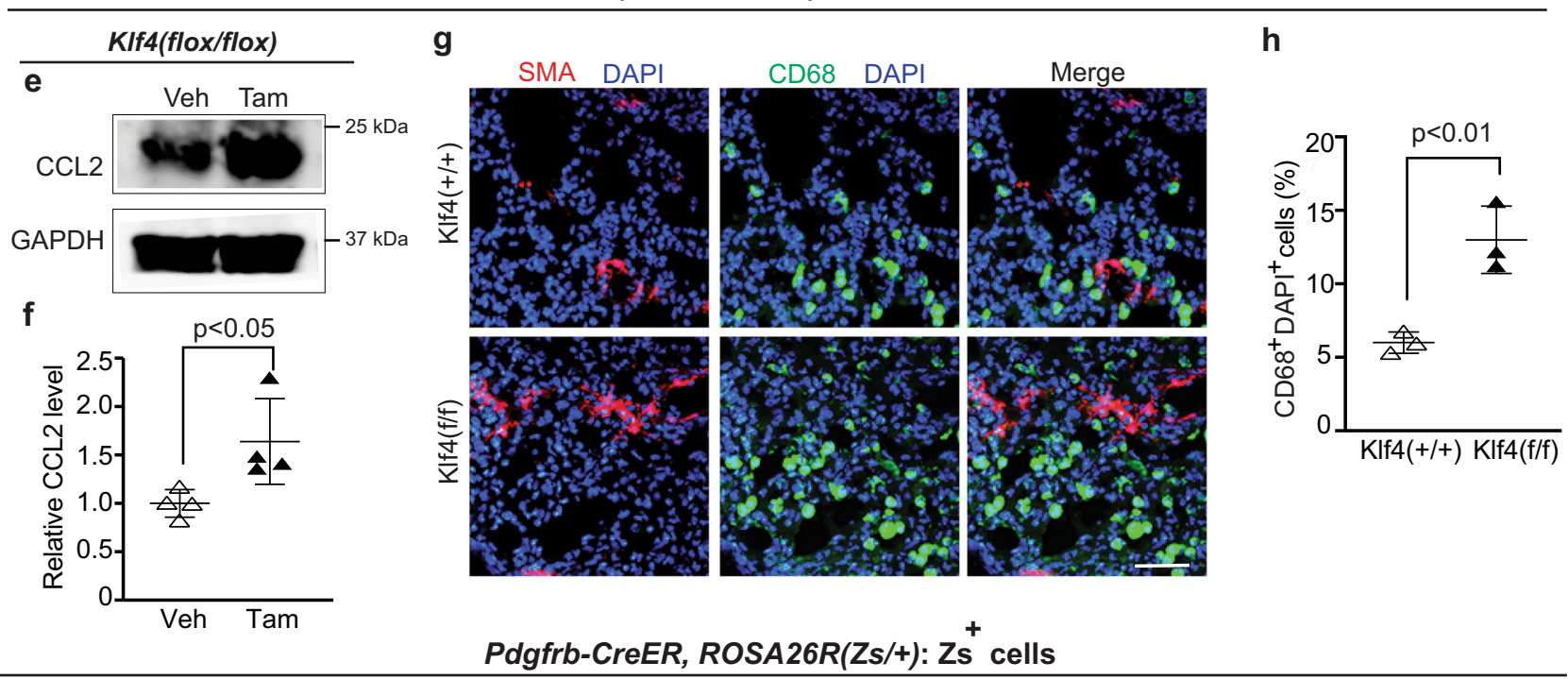

Cx in conditioned medium (CM) from siKIf4 vs. Scr SMCs

(i) siCcr2 vs. Scr;

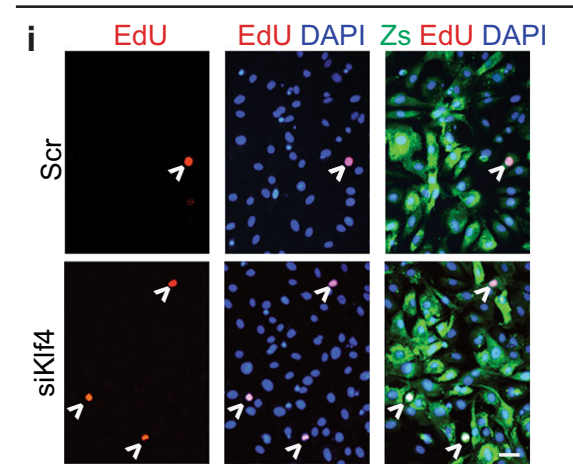

k

(ii) $\mathrm{Cx}$ in $\mathrm{CM}$ from siKIf4 SMCs
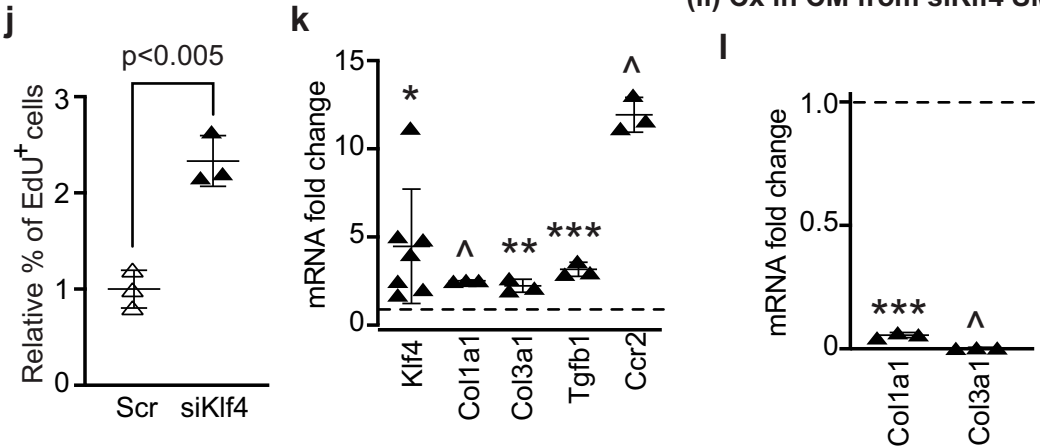

Fig. 6 Reduction of KIf4 in SMA ${ }^{+}$cells increases Ccl2 levels and noncell autonomously induces Ccr2-mediated collagen expression in PDGFR- $\beta^{+}$cells. a-d KIf4 siRNA or scrambled (Scr) RNA treatment of mouse airway SMCs. In $\mathbf{a}$ and $\mathbf{b}$, transcript levels were assayed by $q$ RT-PCR. $n=3$ in triplicate. ${ }^{\star \star}$, KIf4 ( $p=0.0035), \mathrm{Cl} 2(p=0.0013)$, ns (not significant) vs. Scr. Dot blots show CCL2 protein levels in conditioned medium (CM) (c) with densitometry normalized to $\operatorname{Scr}(\mathbf{d}) . n=3, p=0.0010$. e-h The lungs of Acta2-CreER ${ }^{T 2}$ mice carrying KIf4(flox/flox) or KIf4(+/+) were analyzed 14 days after a bleomycin dose. In e and f, mice were induced with vehicle (Veh; corn oil) or tamoxifen (Tam) prior to bleomycin, and lung lysates were subjected to western for $\mathrm{CCL} 2$ and GAPDH with densitometry relative to GAPDH and normalized to vehicle. $n=4, p=0.033$. In $\mathbf{g}$ and $\mathbf{h}$, mice were induced with tamoxifen prior to bleomycin, and lung sections were stained for SMA and macrophage marker CD68. Percent of DAPI + cells that express CD68 was quantified (h). $n=3$ mice, five sections per mice and an average of $906 \mathrm{DAPI}+$ cells per section, $p=0.0073$. i-I Pdgfrb-CreER ${ }^{T 2}, R O S A 26 R^{(Z s /+)}$ mice were induced with tamoxifen, and $\mathrm{Zs}^{+}$cells were isolated by FACS. In i-k, Zs ${ }^{+}$cells were cultured (cx) in CM from airway SMCs pretreated with siKIf4 or Scr RNA. In $\mathbf{i}$, cells were cultured with EdU for $8 \mathrm{~h}$ prior to staining for EdU and nuclei (DAPI) and direct Zs imaging. The percent of DAPI+ cells that were EdU + (arrowheads) was quantified and normalized to percentage for Scr CM-treated cells (j). $n=3$ experiments, five microscopic fields of view per experiment and on average $279 \mathrm{DAPI}^{+}$cells per field, $p=0.0022$. In $\mathbf{k}$, transcript levels relative to Gapdh were measured by qRT-PCR and normalized to Scr CM-treated cells. $n=7$ for KIf4 and $n=3$ for other transcripts. ${ }^{\star} p=0.016 ;{ }^{* \star} p<0.0043 ;{ }^{\star \star \star} p=0.0008 ;{ }^{\wedge},<0.0001 \mathrm{vs}$. Scr. In I, Zs ${ }^{+}$cells were subjected to siCcr2 vs. Scr and then cultured in medium preconditioned by siKlf4-treated SMCs. qRT-PCR was used to measure Col1a1 and Col3a1 mRNA levels. $n=3,{ }^{\star \star \star} p=0.0008$, $\hat{p} p<0.0001$ vs. Scr. Two-tailed Student's $t$ test was used for all analysis. Data are averages \pm SD. Source data are provided as a Source Data file. Scale bars, $50 \mu \mathrm{m}$. 
a

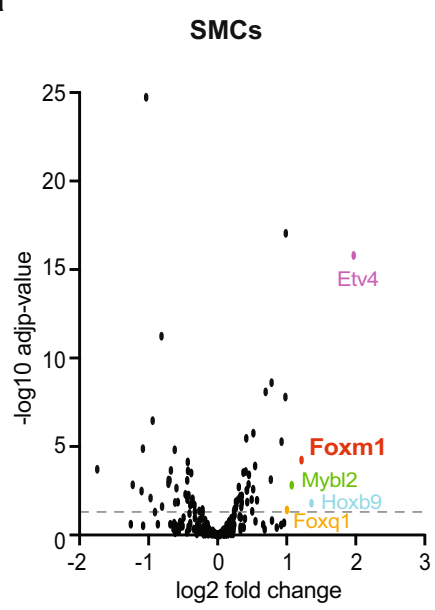

b

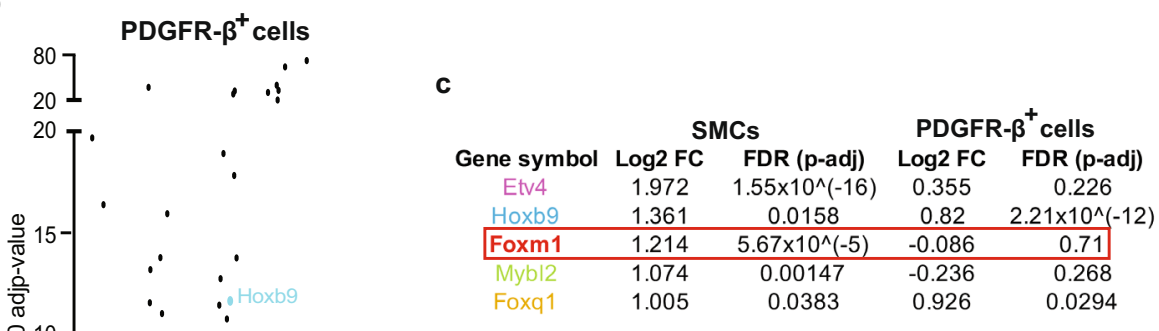

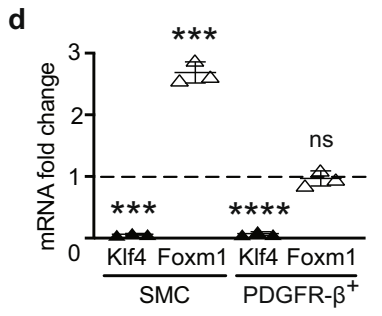

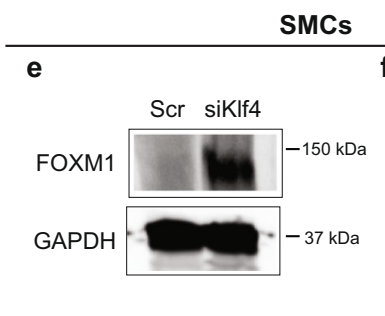

SMCs

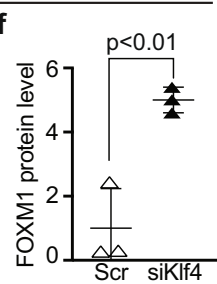

Acta2-CreER, KIf4(flox/flox)

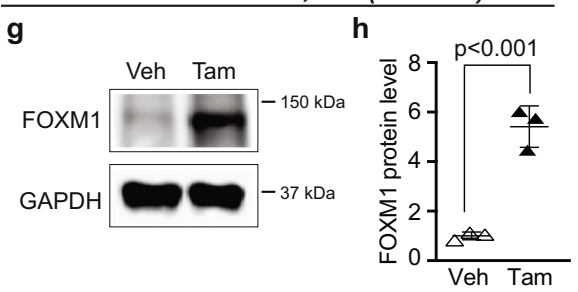

SMCs

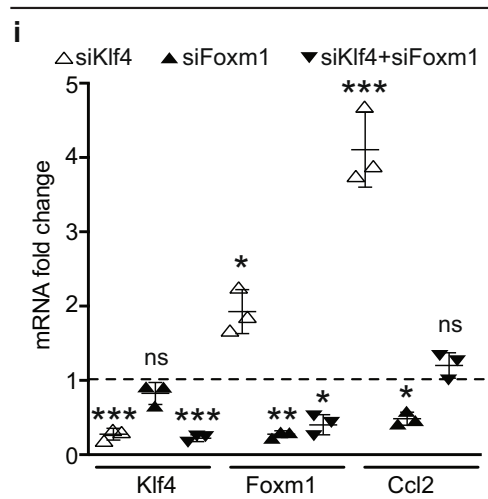

j

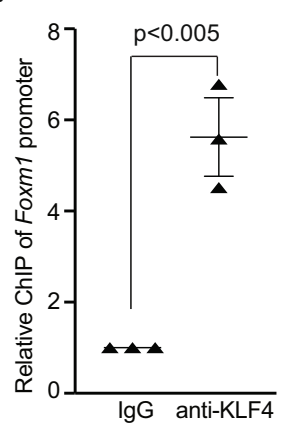

PDGFR- $\beta+$ cells vs. SMCs

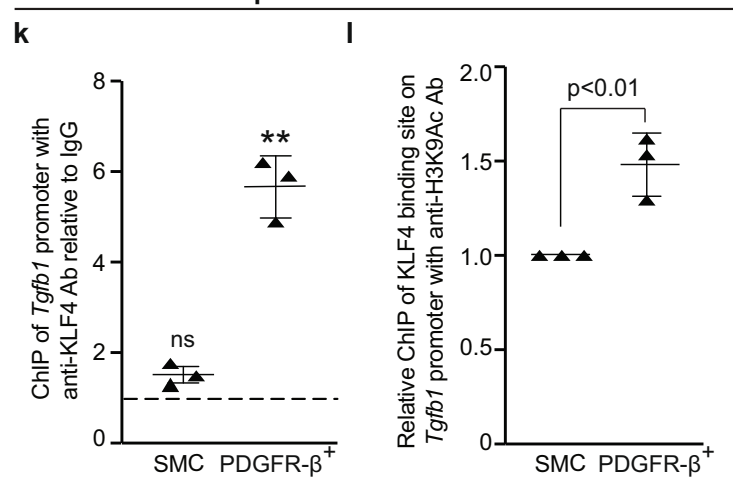

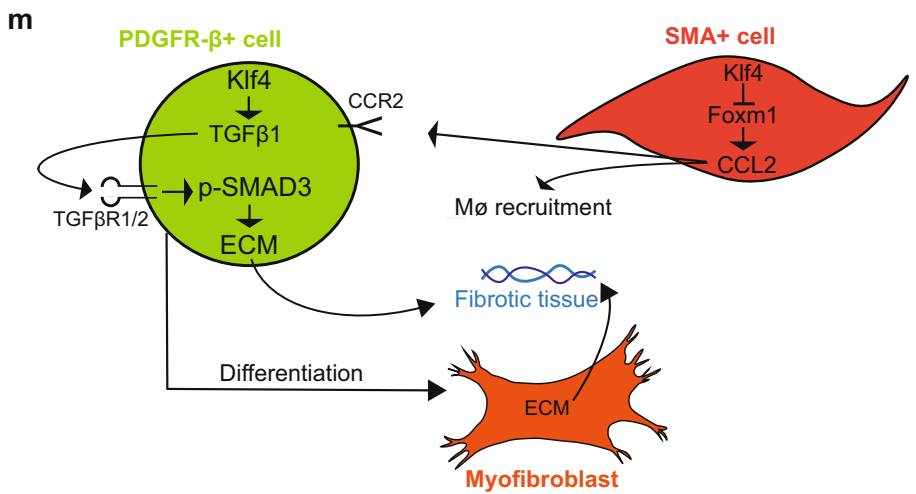

heterodimeric integrins that contain the integrin av subunit, and Pdgfrb-Cre, Itgav(flox/flox) mice are protected against bleomycininduced lung fibrosis ${ }^{36}$. Future investigations into potential interactions of KLF4 and regulatory elements of the integrin av gene in lung mesenchymal cells are likely to be of high yield.

In contrast to our results with PDGFR- $\beta^{+}$cells, KLF4 reduction in $\mathrm{SMA}^{+}$cells of the normal lung, which are predominantly airway and vascular SMCs, exacerbates fibrosis and myofibroblast accumulation in bleomycin-treated mice, but in culture has no effect on levels of Tgfb1, collagens 1a1, 3a1 and fibronectin (Figs. 5 and 6). Yet, this perturbation markedly induces CCL2 levels and accumulation of $\mathrm{CD}^{+} 8^{+}$cells, and CCL2 upregulation has previously been demonstrated in lungs of IPF patients and bleomycin-treated rodents ${ }^{48,60}$. Of note, there is precedence for 
Fig. 7 KLF4 differentially regulates downstream genes in SMCs and PDGFR- $\boldsymbol{\beta}^{+}$cells. Lung PDGFR- $\beta^{+}$cells (Zs ${ }^{+}$cells from Pdgfrb-CreER $\left.{ }^{T 2}, R O S A 26 R^{(Z s /+}\right)$ mice) and SMCs were compared. a-f Cells were treated with KIf4 siRNA or Scr RNA. In a-c, cells were subjected to bulk RNA-seq; $n=3$. Of the TFs predicted with TRANSFAC to bind Ccl2 promoter (see Supplementary Data 3), expression levels of genes present in RNA-seq data are shown (a, b). Among predicted CCl2-binding genes, the five most upregulated genes in SMCs are indicated, and log2 fold change (FC) and false discovery rate (FDR) in SMCs and PDGFR- $\beta^{+}$cells are displayed (c). In d, qRT-PCR was used to assess Klf4 and Foxm1 transcript levels relative to Gapdh and normalized to Scr; $n=3$ in triplicate. ${ }^{\star \star \star}$, KIf4_SMC $(p=0.0004)$, Foxm1_SMC $(p=0.0006)$, Klf4_PDGFR- $\beta^{+}(p<0.0001)$ and ns (not significant) with siKlf4 vs. Scr. In e, f, westerns for FOXM1 and GAPDH with densitometry of their ratio and normalized to Scr; $n=3, p=0.0060$. g, h Acta2-CreER ${ }^{T 2}$, KIf4(flox/flox) mice were induced with vehicle (corn oil) or tamoxifen, and lung lysates were evaluated as in $\mathbf{e}$ and $\mathbf{f}$ with normalization to vehicle; $n=3, p=0.0009$. i SMCs were treated with Scr, siKIf4, siFoxm1 or both siKIf4 and siFoxm1. Transcript levels of Klf4, Foxm1 and Ccl2 relative to Gapdh as assessed by qRT-PCR and normalized to Scr; $n=3$. ${ }^{\star}$, siKlf4_Foxm1 $(p=0.012)$, si-both_Foxm1 $(p=0.012)$, siFoxm1_Ccl2 $(p=0.023)$; ${ }^{\star \star}$, siFoxm1_Foxm1 ( $\left.p=0.0036\right)$; ${ }^{\star \star \star}$, siKIf4_KIf4 ( $p=0.0003)$, si-both_KIf4 ( $p<0.0001)$, siKIf4_Ccl2 ( $p=0.0007)$; ns vs. Scr. j ChIP assay for SMCs using anti-KLF4 antibody or control IgG, followed by qPCR with primers for Foxm1 promoter. Signal for coprecipitated DNA with anti-KLF4 antibody normalized to IgG is shown; $n=3, p=0.0021$. k, I ChIP was performed in SMCs and PDGFR- $\beta^{+}$cells using QPCR primers for Tgfb1 promoter. In $\mathbf{k}$, anti-KLF4 antibody or IgG was used for pulldown and coprecipitated DNA with anti-KLF4 antibody is relative to IgG. $n=3$, SMC (ns) and PDGFR- $\beta^{+}(p=0.0071)$ with anti-KLF4 antibody vs. IgG. In I, lysates were subjected to immunoprecipitation with anti-H3K9Ac antibody and amount of coprecipitated DNA from each cell type is relative to PCR product amplified from input samples reserved before immunoprecipitation. This ratio for PDGFR- $\beta^{+}$cells is normalized to SMCs; $n=3, p=0.0076$. Two-tailed Student's $t$ test was used for analysis $(\mathbf{d}, \mathbf{f}, \mathbf{h}-\mathbf{I})$. $\mathbf{m}$ Mesenchymal cell-type-specific KLF4 effects in lung fibrosis. Data are averages \pm SD. Source data are provided as a Source Data file.

SMCs secreting chemokines and cytokines during the inflammatory response ${ }^{61,62}$. We found that conditioned medium from SMCs subjected to Klf4 knockdown induces PDGFR- $\beta^{+}$cells to proliferate and express increased levels of Klf4, Ccr2, Tgfb1 and collagens $1 \mathrm{a} 1$ and $3 \mathrm{a} 1$, and the collagen upregulation is Ccr2 dependent. In the context of bleomycin administration, lung hydroxyproline content is reduced by global Ccr 2 deletion or gene transfer of a dominant negative $C c l 2^{48,63}$. A trial of carlumab, an inhibitor that binds and neutralizes profibrotic activities of CCL2, unfortunately showed no benefit in IPF patients and in fact a trend to worse outcomes ${ }^{64}$. Surprisingly, however, carlumab treatment was associated with an increase-not the expected decrease-in free CCL2 serum levels ${ }^{64}$, suggesting that alternate means to reduce CCL2-CCR2 signaling remains a potentially viable therapeutic approach.

Thus, a relevant mechanistic question arises: how does KLF4 induce markedly distinct effects in PDGFR- $\beta^{+}$cells and SMCs? Among TFs bioinformatically predicted to bind within the $5-\mathrm{kb}$ region upstream of $\mathrm{Ccl} 2$, our unbiased transcriptomic screen of Klf4 silenced cells identified Foxm1 as one of the few most upregulated genes in SMCs that is not also upregulated in PDGFR- $\beta^{+}$cells (Fig. 7). Klf4 has been shown to negatively regulate Foxm 1 in a gastrointestinal cancer cell line, and FOXM1 induces CCL2 levels in hepatocytes ${ }^{50,52}$. Our studies demonstrate a link in lung SMCs between Klf4 downregulation to upregulation of FOXM1 and in turn, increased CCL2. Interestingly, no such Klf4-mediated regulation of Foxm1 or $\mathrm{Ccl} 2$ was observed in PDGFR- $\beta^{+}$cells. In contrast, Klf4 silencing results in Tgfb1 levels that are increased in PDGFR- $\beta^{+}$cells but unaltered in SMCs. These differential effects are likely due to the increased accessibility (as indicated by histone $\mathrm{H} 3 \mathrm{~K} 9$ acetylation) and hence binding of KLF4 to the Tgfbl promoter in PDGFR- $\beta^{+}$cells.

Taken together, our results indicate that KLF4 is a major player in the lung mesenchyme during fibrosis, having opposing celltype-specific effects: profibrotic in PDGFR- $\beta^{+}$cells and antifibrotic in $\mathrm{SMA}^{+}$cells. This work also highlights the importance of studying intercellular signaling between mesenchymal cells and their transitions during the pathogenesis of lung fibrosis. Further studies of the role of diverse mesenchymal cell types in the course of lung fibrosis are warranted and promise to advance novel therapeutic strategies for this lethal disease.

\footnotetext{
Methods

Animals. C57BL/6 wild-type mice and Cre reporter ROSA26R(ZsGreen1/ZsGreen1), ROSA26R ${ }^{(m T m G / m T m G)}$ and $R O S A 26 R^{(Y F P / Y F P)}$ mice were obtained from Jackson
}

Laboratory ${ }^{6-67}$, and $\mathrm{Klf4} 4$ (flox/flox) mice were from purchased from the Mutant Mouse Resource \& Research Center ${ }^{68}$. Acta2-CreER ${ }^{T 2}$, Pdgfrb-CreER ${ }^{T 2}$ and the multicolor ROSA26R $(R b / R b)$ mice were also used $24,39,69,70$. Experiments included male and female mice aged 3-4 months and sex and age-matched controls. Mice are housed in rooms with a $12 \mathrm{~h}$ light $/ 12 \mathrm{~h}$ dark cycle, ambient temperature of $69-71^{\circ} \mathrm{F}$ and $38-50 \%$ humidity.

Tamoxifen, bleomycin and hypoxia treatment. For fate mapping, clonal analysis and cell isolation, mice were injected intraperitoneally with tamoxifen ( $1 \mathrm{mg}$ or $\sim 40 \mathrm{mg} / \mathrm{kg}$ body weight per day for 5 days). For in vivo Klf4 deletion, mice were injected with tamoxifen ( $1 \mathrm{mg}$ or $\sim 40 \mathrm{mg} / \mathrm{kg}$ per day for 10 days) or with equivalent volume of corn oil (vehicle). After tamoxifen injections, mice were rested for 5 days before bleomycin (Sigma-Aldrich) or hypoxia treatment. Bleomycin was administered orotracheally as a single dose of $1.5 \mathrm{U} / \mathrm{kg}$ body weight. As a control for bleomycin treatment, a similar volume of PBS was given orotracheally. Mice were euthanized and lungs were harvested 5, 7, 14 or 21 days after bleomycin or PBS administration. Alternatively, mice were exposed to normoxia or hypoxia $\left(\mathrm{FiO}_{2}\right.$ $10 \%$ ) for 21 days in a rodent hypoxia chamber with a calibrated oxygen controller and sensor (BioSpherix) and then euthanized, and lungs were harvested.

Lung preparation and immunohistochemistry. To prepare for sectioning and cell isolation, after euthanizing mice, the pulmonary vasculature was flushed by injecting PBS through the right ventricle. For vibratome sections, lungs were inflated by tracheal injection of $2 \%$ low-melting agarose through the trachea. Lungs were harvested, incubated in Dent's fixative ( $4: 1$ methanol/dimethyl sulfoxide) overnight at $4{ }^{\circ} \mathrm{C}$ and then stored in $100 \%$ methanol at $-20^{\circ} \mathrm{C}$. In preparation for staining, lungs were bleached in $5 \% \mathrm{H}_{2} \mathrm{O}_{2}$ in methanol, rehydrated sequentially in $75 \%, 50 \%$ and $25 \%$ and $0 \%$ methanol in PBS. A vibratome was used to cut $150 \mu \mathrm{m}$ thick sections. For cryosections, flushed lungs were harvested and fixed in $4 \%$ paraformaldehyde (PFA) overnight, washed and incubated in 30\% sucrose. Lungs were then embedded in optical cutting temperature compound (Tissue-Tek), frozen and stored at -20 or $-80^{\circ} \mathrm{C}$. A cryostat was used to cut $10-30 \mu \mathrm{m}$ thick sections. Both vibratome sections and cryosections were blocked in $0.1 \%$ Triton $\mathrm{X}-100$ in PBS (PBS-T) containing 5\% goat serum, washed with PBS-T and incubated with primary antibodies overnight at $4{ }^{\circ} \mathrm{C}$. On the next day, sections were washed with PBS-T, incubated in secondary antibodies for $2 \mathrm{~h}$, washed again with PBS-T and mounted in fluorescence mounting medium (DAKO).

Lungs from human adult IPF patients and age-matched controls were fixed in formalin, paraffin embedded and sectioned. Sections were deparaffinized with xylene and rehydrated into water through serial dilutions of ethanol. Rehydrated sections were washed with TNT $(10 \mathrm{mM}$ Tris- $\mathrm{HCl}, \mathrm{pH} 8.0,150 \mathrm{mM} \mathrm{NaCl}, 0.2 \%$ Tween-20) for $10 \mathrm{~min}$. Sections were then incubated in prewarmed $\left(65^{\circ} \mathrm{C}\right)$ antigen retrieval buffer for $30 \mathrm{~min}$ in a microwave per manufacturer's instructions (DAKO). After cooling to room temperature, sections were washed in TNT buffer for $10 \mathrm{~min}$, blocked in PBS-T containing $5 \%$ goat serum for $30 \mathrm{~min}$ and immunostained as described above for mouse sections.

Primary antibodies used were chicken anti-GFP (1:100, Abcam), rabbit antiKLF4 (1:100-200, Cell Signaling), rat anti-CD68 (1:200, Bio-Rad), rat antiMECA32 (1:15, Developmental Studies Hybridoma Bank), directly conjugated Cy3 anti-SMA (1:150-250, Sigma-Aldrich), and biotinylated anti-PDGFR- $\beta$ (1:50, $\mathrm{R} \& \mathrm{D})$. Elite $A B C$ reagents (Vector Laboratories) and fluorescein tyramide system (PerkinElmer) were used to amplify the biotinylated PDGFR- $\beta$ staining ${ }^{24}$. Secondary antibodies (1:250-500) were raised in goat and conjugated to either Alexa-488 (anti-chicken [Abcam], anti-rabbit [Invitrogen]), -564, (anti-rabbit, 
anti-rat [Invitrogen]), or -647 fluorophore (anti-rabbit [Invitrogen]). Nuclei were visualized by DAPI (1:1000, Sigma-Aldrich).

In the bleomycin model, lung fibrosis is patchy and thus, most sections include both highly fibrotic and non/low fibrotic areas. Fibrotic lung regions were considered SMA+ areas in the parenchyma that were distant from airways or blood vessels. These regions were generally hypercellular (as evidenced by staining with DAPI) and had distorted alveolar structures. In contrast, nonfibrotic regions had relatively intact alveoli, little or no SMA + cell accumulation in the parenchyma (beyond airway or vascular SMCs) and were not hypercellular.

Lung collagen content. For qualitative analysis of collagen content, the Picrosirius Red Stain Kit was used per the instructions of the manufacturer (Abcam). Lung cryosections $(10 \mu \mathrm{m})$ were hydrated in distilled water, incubated in Picrosirius red solution for $1 \mathrm{~h}$, rinsed twice in acetic acid solution, dehydrated in absolute alcohol and mounted in DPX mounting medium (Sigma-Aldrich). For quantitative analysis, Sircol Collagen Assay was performed according to the instructions and with buffers supplied by the manufacturer (Biocolor). Briefly, the entire right lung was disrupted using TissueLyser II (Qiagen), digested overnight in pepsin and acetic acid and centrifuged at $18,400 \times \mathrm{g}$ for $30 \mathrm{~min}$. The supernatant was collected, and $1 \mathrm{ml}$ Sircol dye was incubated with $100 \mu \mathrm{l}$ of the supernatant for $1 \mathrm{~h}$. This sample was centrifuged at $13,500 \times \mathrm{g}$ for $10 \mathrm{~min}$, and the resulting pellet was washed in the acid salt wash buffer and resuspended in $250 \mu \mathrm{l}$ alkali buffer. Optical density (OD) at $555 \mathrm{~nm}$ was measured, and the collagen concentration was determined by comparing the OD of samples with that of standards of known collagen concentrations.

Fate mapping. Mice carrying an inducible Cre recombinase (Pdgfrb-CreER ${ }^{T 2}$ or Acta2-CreER $\left.R^{T 2}\right)$ and a Cre reporter [ROSA26R ${ }^{(m T m G /+)}, R O S A 26 R^{(Z s /+)}$ or $\left.R O S A 26 R^{(Y F P /+)}\right]$ were injected with tamoxifen, rested and a single orotracheal dose of PBS (control group) or bleomycin (experimental group) was administered. On day 7 or 14 after orotracheal treatment, mice were euthanized and lungs were harvested. Lung sections were stained for SMA and nuclei (DAPI) as well as with an antibody directed against GFP or directly imaged for Zs. The contribution of different lineages to the myofibroblast pool were determined by scoring the percentage of parenchymal $\mathrm{SMA}^{+} \mathrm{DAPI}^{+}$cells (excluding vascular and airway SMCs) that were also marked by the Cre reporter.

Clonal analysis. Pdgfrb-CreER $R^{T 2}, R O S A 26 R^{(R b /+)}$ mice were injected with tamoxifen, rested and then exposed to a single orotracheal dose of PBS or bleomycin. Fourteen days later, lungs were harvested. Cryosections were stained with DAPI, and $\mathrm{Rb}$ colors (i.e., Cerulean, mOrange, and mCherry) were directly imaged. The number of contiguous cells of the same $\mathrm{Rb}$ color was scored to determine the clonal patch size. Patch area of congruent cells marked with the same fluorophore were determined using Image J software.

Cell proliferation in the murine lung. $P d g f r b-C r e E R^{T 2}, R O S A 26 R^{(Y F P /+)}$ mice that were also either Klf4 4 (floxfflox) or wild type for Klf4 were injected with tamoxifen, rested, subjected to orotracheal bleomycin and euthanized 5 or 14 days thereafter. Twelve hours prior to euthanasia, mice were injected intraperitoneally with $2.5 \mathrm{mg}$ of EdU (Thermo Fisher Scientific). Harvested lungs were fixed in 4\% PFA overnight, permeabilized in $0.5 \%$ PBS-T for 30 min, stained with the Click-iT EdU Alexa Fluor 555 Imaging Kit per instructions of the manufacturer (Thermo Fisher Scientific) and then costained for YFP and nuclei (DAPI). Per each genotype analyzed, the percent of $\mathrm{YFP}^{+}$cells that express EdU was determined.

Isolation of lung PDGFR- $\boldsymbol{\beta}^{+}$and SMA ${ }^{+}$cells. Lungs were finely minced and incubated in $2 \mathrm{mg} / \mathrm{ml}$ collagenase I (Worthington) in PBS at $37^{\circ} \mathrm{C}$ for $20 \mathrm{~min}$. The digestion mixture was passed through a 14-gauge pipetting needle (Cadence Science), incubated at $37^{\circ} \mathrm{C}$ for an additional $20 \mathrm{~min}$ and then filtered through a $70 \mu \mathrm{m}$ cell strainer (Falcon). This sc suspension filtrate was centrifuged at $340 \times g$ for $5 \mathrm{~min}$, and the pellet was resuspended in cold PBS with $1 \%$ fetal bovine serum (FBS; Invitrogen). The resuspended cells were incubated with either PE-conjugated anti-PDGFR- $\beta$ antibody (1:200, Miltenyi Biotec) for $20 \mathrm{~min}$ in the case of Pdgfrb$\mathrm{CreER}^{T 2}, \mathrm{Klf} 4$ (flox/flox) mice or DAPI (1:1000) to label dead cells for $10 \mathrm{~min}$ in the case of $P d g f r b-C r e E R^{T 2}, R O S A 26 R^{(Z s /+)}$ mice. The sample was then washed in cold PBS with $1 \%$ FBS and centrifuged at $340 \times g$ for 5 min. The resulting pellet was resuspended in PBS containing 1\% FBS and 0.02\% EDTA. DAPI (1:2000) was added to cells from Acta2-CreER ${ }^{T 2}, R O S A 26 R^{(Z s /+)}$ mice or cells from Pdgfrb-

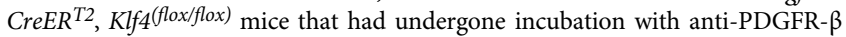
antibody. Finally, $\mathrm{Zs}^{+}{ }^{+} \mathrm{DAPI}^{-}$or PDGFR- $\beta^{+} \mathrm{DAPI}^{-}$cells were sorted on a BD FACSAria II cell sorter. Cells stained with DAPI alone were used as a control for anti-PDGFR- $\beta$ antibody specificity. Lungs from C57BL/6 mice were processed similarly to those of $P d g f r b-C r e E R^{T 2}, R O S A 26 R^{(Z s /+)}$ mice, and isolated cells were used as a control for auto-fluorescence in the Zs channel. FlowJo was used to analyze data.

Western blot. Lung tissues or cells were lysed in RIPA buffer containing complete protease inhibitor and PhosSTOP phosphatase inhibitor cocktails (Roche Applied
Science). Lysate was centrifuged at $13,500 \times g$ for $10 \mathrm{~min}$ at $4{ }^{\circ} \mathrm{C}$, and the supernatant was collected. Protein concentrations were determined by the BCA assay (Pierce). Laemmli buffer (Bio-Rad) containing $\beta$-mercaptoethanol was added to $40 \mu \mathrm{g}$ protein, and boiled at $95^{\circ} \mathrm{C}$ for $5 \mathrm{~min}$. Protein were separated by SDS-PAGE, transferred to nitrocellulose membranes (Millipore) overnight, blocked with 5\% bovine serum albumin in PBS containing 0.05\% Tween-20 for $1 \mathrm{~h}$ and incubated with primary antibody overnight at $4{ }^{\circ} \mathrm{C}$. Primary antibodies used were: rabbit antiKLF4, rabbit anti-SMAD2/3, rabbit anti-GAPDH (1:500, 1:500, 1:5000; Cell Signaling Technology), rabbit anti-phospho-SMAD3, rabbit anti-collagen 1, rabbit anti-CCL2, rabbit anti-FOXM1 (1:500, 1:1000, 1:1000, 1:1000; Abcam), mouse anti-SMA (1:2000, Sigma) and mouse anti-fibronectin (1:5000, BD Biosciences). After washing, membranes were incubated with horseradish peroxidase-conjugated secondary antibodies (1:2000; DAKO) for $1 \mathrm{~h}$. Detection was performed with the Western Blotting Substrate Plus (Pierce) and GBOX imaging system (Syngene).

Cell culture. Cells were cultured on dishes coated with $0.1 \%$ gelatin, and cells of passages 2-4 were used for experiments. $\mathrm{Zs}^{+}$cells isolated from the lungs of Pdgfrb-CreER ${ }^{T 2}$, ROSA26R $(Z s /+)$ mice were cultured in low glucose DMEM (Gibco) supplemented with $10 \% \mathrm{FBS}, 1 \%$ penicillin/streptomycin (Life Technologies), $5 \mu \mathrm{g} /$ $\mathrm{ml}$ gentamycin (Sigma-Aldrich) and $4 \mu \mathrm{g} / \mathrm{ml}$ amphotericin B (Corning ${ }^{\mathrm{TM}}$ ). Prior to staining, $\mathrm{Zs}^{+}$cells were fixed in $4 \% \mathrm{PFA}$ and permeabilized in $0.5 \%$ Triton X-100 in PBS. Primary mouse airway SMCs (Cell Biologics) were cultured in SMC medium (Cell Biologics) supplemented with EGF and FGF, 10\% FBS and 1\% penicillin/streptomycin.

siRNA-mediated knockdown of Klf4. Murine lung PDGFR- $\beta^{+}$cells or airway SMCs were serum starved for $2 \mathrm{~h}$ and then transfected with Lipofectamine RNAiMAX or Lipofectamine 2000 (Invitrogen) containing siRNAs (Origene or Dharmacon) targeted against Klf4 $(50 \mathrm{nM})$ or Scr RNA for $6 \mathrm{~h}$. Cells were washed in PBS and cultured in supplemented SMC medium for $72 \mathrm{~h}$. The conditioned medium was then collected, and cells were washed in PBS. Cell lysates were subjected to western blot or RNA was isolated for qRT-PCR analysis.

Conditioned medium experiments. Conditioned medium from Scr RNA or siKlf4-treated SMCs was centrifuged at $940 \times g$ for $5 \mathrm{~min}$, and the supernatant was collected. Isolated $\mathrm{Zs}^{+}$cells from the lungs of Pdgfrb-CreER ${ }^{T 2}, R O S A 26 R^{(Z s /+)}$ mice were serum starved for $2 \mathrm{~h}$, washed two times in PBS and incubated with the conditioned medium supernatant for $24 \mathrm{~h}$ prior to collection for qRT-PCR, dot blot or proliferation assay. For proliferation studies, during the last $8 \mathrm{~h}$ of this incubation, EdU $(10 \mu \mathrm{m})$ from the Click-iT EdU Alexa Fluor 555 Imaging Kit (Thermo Fisher Scientific) was added to the conditioned medium supernatant. Cells were then fixed in 4\% PFA overnight, permeabilized in $0.5 \%$ Triton X-100 in PBS and costained for EdU and other proteins per the manufacturer's instructions.

Quantitative real-time PCR analysis. Cells were lysed, and RNA was isolated per the PureLink ${ }^{\mathrm{TM}}$ RNA Mini Kit (Invitrogen). The iScript cDNA Synthesis Kit (Bio$\mathrm{Rad}$ ) was used for reverse transcription of $200 \mathrm{ng}$ RNA. The mRNA levels of selected genes were determined by qRT-PCR and normalized to Gapdh. Forward and reverse primer pairs for the assayed genes are listed in Supplementary Table 1.

Dot blot. Dot blot apparatus was assembled with nitrocellulose membrane and filter paper, following instructions of the manufacturer (Bio-Rad). SMC conditioned medium supernatant $(100 \mu \mathrm{l})$ was applied to each Dot blot well, and the nitrocellulose membrane was allowed to dry for $1 \mathrm{~h}$. The membrane was then blocked in $5 \%$ dry milk in tris-buffered saline (TBS) containing $0.1 \%$ Tween-20, and incubated in rabbit anti-CCL2 antibody (1:1000, Abcam) overnight at $4{ }^{\circ} \mathrm{C}$. The next day, after washing in TBS with $0.1 \%$ Tween-20, the membrane was incubated with horseradish peroxidase-conjugated anti-rabbit secondary antibody (1:2000; DAKO) for $1 \mathrm{~h}$. Detection was performed with the Western Blotting Substrate Plus (Pierce) and GBOX imaging system (Syngene).

Chromatin immunoprecipitation. Eight confluent $10-\mathrm{cm}$ dishes of PDGFR- $\beta^{+}$ cells or SMCs $\left(\sim 8 \times 10^{6}\right.$ cells $)$ were treated with $1 \%$ formaldehyde for $10 \mathrm{~min}$ to cross-link protein to DNA and were neutralized with glycine. Cells were scraped in cold PBS and subjected to ChIP assay using the SimpleChIP Plus Enzymatic Chromatin IP Kit (Cell Signaling) with an antibody targeting KLF4 (Abcam) or Histone H3K9Ac (Cell Signaling). Coprecipitated DNA was analyzed by qPCR with primer sets (sense and antisense) targeting the promoter locus of Foxm 1 (5' GCCGATTGGCGACGCT-3' and 5'-CGCCGCTTTCAGTTGTTCCG-3') or Tgfb1 (5'-CCTTGACACTCTCATCCGCA- $3^{\prime}$ and $5^{\prime}$-TGGGACTTCGTGAGAAAACA GA-3'). For ChIP assay with the anti-KLF4 antibody, the amount of coprecipitated DNA was normalized to that from ChIP using control rabbit IgG (1:500, Cell Signaling). For ChIP assay with the H3K9Ac antibody, the amount of coprecipitated DNA in PDGFR- $\beta^{+}$cells is relative to the amount of PCR product amplified from input samples reserved before immunoprecipitation and then normalized to this value in SMCs. 
Imaging. Images were acquired with Leica SP5 or SP8 confocal microscope, PerkinElmer UltraView Vox Spinning Disc confocal microscope or Nikon Eclipse 80i upright fluorescent microscope. For image processing, analysis and cell counting, Volocity software (PerkinElmer), Image J and Adobe Photoshop were used.

Statistical analysis. Student's $t$ test, one-way ANOVA with Tukey's multiple comparisons test were used to analyze the data (Prism 7 software). Statistical significance threshold was set at $p \leq 0.05$. Tests assumed normal distribution and were two-sided, and all data are presented as mean \pm standard deviation.

Study approval. All mouse experiments were approved by the IACUC at Yale University and were performed in accordance with relevant ethical regulations. All procedures involving human subjects were approved by the Institutional Review Board of Yale University (IRB\# 1307012431), and we complied with all relevant ethical regulations. Written informed consent was obtained from all participants and/or a family member prior to inclusion in the study.

Reporting summary. Further information on research design is available in the Nature Research Reporting Summary linked to this article.

\section{Data availability}

Data are available in the main figures, Supplementary Figures and Supplementary Data. The source data underlying Figs. 1c, d, f, h, i, 2b, d, e, g, i, 3c-e, g, i, 4a-h, 5c-e, g, i, 6a-f, h, j-l and 7d-l and Supplementary Figs. 5b, 8, 9b, c, 11a, c, 13a, c, 14a, b and 15b are provided as a Source Data file. The TRANSFAC database is publicly available (https:// portal.genexplain.com/cgi-bin/portal/login.cgi). The bulk and scRNA-seq data generated in this study have been deposited in the Gene Expression Omnibus database under accession code GSE184672. Source data are provided with this paper.

Received: 17 November 2019; Accepted: 19 November 2021; Published online: 10 December 2021

\section{References}

1. Todd, N. W., Luzina, I. G. \& Atamas, S. P. Molecular and cellular mechanisms of pulmonary fibrosis. Fibrogenes. Tissue Repair 5, 11-11 (2012).

2. Blackwell, T. S. et al. Future directions in idiopathic pulmonary fibrosis research. An NHLBI workshop report. Am. J. Respir. Crit. Care Med. 189, 214-222 (2014).

3. Kropski, J. A. \& Blackwell, T. S. Progress in understanding and treating idiopathic pulmonary fibrosis. Annu. Rev. Med. 70, 211-224 (2019).

4. Martinez, F. J. et al. The clinical course of patients with idiopathic pulmonary fibrosis. Ann. Intern. Med. 142, 963-967 (2005).

5. Ley, B., Collard, H. R. \& King, T. E. Clinical course and prediction of survival in idiopathic pulmonary fibrosis. Am. J. Respir. Crit. Care Med. 183, 431-440 (2011).

6. Richeldi, L. et al. Efficacy and safety of nintedanib in idiopathic pulmonary fibrosis. N. Engl. J. Med. 370, 2071-2082 (2014).

7. Sheikh, A. Q., Lighthouse, J. K. \& Greif, D. M. Recapitulation of developing artery muscularization in pulmonary hypertension. Cell Rep. 6, 809-817 (2014).

8. Moore, M. W. \& Herzog, E. L. Regulation and relevance of myofibroblast responses in idiopathic pulmonary fibrosis. Curr. Pathobiol. Rep. 1, 199-208 (2013).

9. Barkauskas, C. E. \& Noble, P. W. Cellular mechanisms of tissue fibrosis. 7. New insights into the cellular mechanisms of pulmonary fibrosis. Am. J. Physiol. Cell Physiol. 306, C987-996 (2014).

10. Phan, S. H. The myofibroblast in pulmonary fibrosis. Chest 122, 286S-289S (2002).

11. Moeller, A., Ask, K., Warburton, D., Gauldie, J. \& Kolb, M. The bleomycin animal model: a useful tool to investigate treatment options for idiopathic pulmonary fibrosis? Int. J. Biochem. Cell Biol. 40, 362-382 (2008).

12. Liu, T., De Los Santos, F. G. \& Phan, S. H. The bleomycin model of pulmonary fibrosis. Methods Mol. Biol. 1627, 27-42 (2017).

13. El Agha, E. et al. Two-way conversion between lipogenic and myogenic fibroblastic phenotypes marks the progression and resolution of lung fibrosis. Cell Stem Cell 20, 261-273 e263 (2017).

14. Zepp, J. A. et al. Distinct mesenchymal lineages and niches promote epithelial selfrenewal and myofibrogenesis in the lung. Cell 170, 1134-1148 e1110 (2017).

15. Kramann, R. et al. Perivascular Gli1+ progenitors are key contributors to injury-induced organ fibrosis. Cell Stem Cell 16, 51-66 (2015).

16. Hung, C. et al. Role of lung pericytes and resident fibroblasts in the pathogenesis of pulmonary fibrosis. Am. J. Respir. Crit. Care Med. 188, 820-830 (2013).

17. Rock, J. R. et al. Multiple stromal populations contribute to pulmonary fibrosis without evidence for epithelial to mesenchymal transition. Proc. Natl Acad. Sci. USA 108, E1475-1483 (2011).
18. He, L. et al. Single-cell RNA sequencing of mouse brain and lung vascular and vessel-associated cell types. Sci. Data 5, 180160 (2018)

19. Kishi, M. et al. Blockade of platelet-derived growth factor receptor-beta, not receptor-alpha ameliorates bleomycin-induced pulmonary fibrosis in mice. PLoS ONE 13, e0209786 (2018).

20. Takahashi, K. \& Yamanaka, S. Induction of pluripotent stem cells from mouse embryonic and adult fibroblast cultures by defined factors. Cell 126, 663-676 (2006).

21. Jopling, C., Boue, S., \& Belmonte, J.C.I. Dedifferentiation, transdifferentiation and reprogramming: three routes to regeneration. Nat. Rev. Mol. Cell Biol. 12, 79-89 (2011).

22. Chambers, I. \& Tomlinson, S. R. The transcriptional foundation of pluripotency. Development 136, 2311-2322 (2009).

23. Jean, J. C. et al. Transcription factor Klf4, induced in the lung by oxygen at birth, regulates perinatal fibroblast and myofibroblast differentiation. PLoS ONE 8, e54806 (2013).

24. Sheikh, A. Q., Misra, A., Rosas, I. O., Adams, R. H. \& Greif, D. M. Smooth muscle cell progenitors are primed to muscularize in pulmonary hypertension. Sci. Transl. Med. 7, 308 ral59 (2015).

25. Ghaleb, A. M. \& Yang, V. W. Kruppel-like factor 4 (KLF4): what we currently know. Gene 611, 27-37 (2017).

26. Murgai, M. et al. KLF4-dependent perivascular cell plasticity mediates premetastatic niche formation and metastasis. Nat. Med. 23, 1176-1190 (2017).

27. Deaton, R. A., Gan, Q. \& Owens, G. K. Sp1-dependent activation of KLF4 is required for PDGF-BB-induced phenotypic modulation of smooth muscle. Am. J. Physiol. Heart Circ. Physiol. 296, H1027-1037 (2009).

28. Davis-Dusenbery, B. N. et al. down-regulation of Kruppel-like factor-4 (KLF4) by microRNA-143/145 is critical for modulation of vascular smooth muscle cell phenotype by transforming growth factor-beta and bone morphogenetic protein 4. J. Biol. Chem. 286, 28097-28110 (2011).

29. Zhang, Y. et al. Kruppel-like factor 4 transcriptionally regulates TGF-betal and contributes to cardiac myofibroblast differentiation. PLOS ONE 8, e63424 (2013).

30. Ke, B., Zhang, A., Wu, X. \& Fang, X. The role of Kruppel-like factor 4 in renal fibrosis. Front. Physiol. 6, 327 (2015).

31. $\mathrm{Hu}, \mathrm{B}$. et al. Gut-enriched Kruppel-like factor interaction with Smad3 inhibits myofibroblast differentiation. Am. J. Respir. Cell Mol. Biol. 36, 78-84 (2007).

32. Phan, S. H., Varani, J. \& Smith, D. Rat lung fibroblast collagen metabolism in bleomycin-induced pulmonary fibrosis. J. Clin. Investig. 76, 241-247 (1985).

33. Lin, L. et al. Krupple-like-factor 4 attenuates lung fibrosis via inhibiting epithelial-mesenchymal transition. Sci. Rep. 7, 15847 (2017).

34. Kapanci, Y., Burgan, S., Pietra, G. G., Conne, B. \& Gabbiani, G. Modulation of actin isoform expression in alveolar myofibroblasts (contractile interstitial cells) during pulmonary hypertension. Am. J. Pathol. 136, 881-889 (1990)

35. Chen, Y. F. et al. Atrial natriuretic peptide-dependent modulation of hypoxiainduced pulmonary vascular remodeling. Life Sci. 79, 1357-1365 (2006).

36. Henderson, N. C. et al. Targeting of alphav integrin identifies a core molecular pathway that regulates fibrosis in several organs. Nat. Med. 19, 1617-1624 (2013).

37. Tsukui, T. et al. Collagen-producing lung cell atlas identifies multiple subsets with distinct localization and relevance to fibrosis. Nat. Commun. 11, 1920 (2020).

38. Xie, T. et al. Single-cell deconvolution of fibroblast heterogeneity in mouse pulmonary fibrosis. Cell Rep. 22, 3625-3640 (2018)

39. Misra, A. et al. Integrin beta 3 regulates clonality and fate of smooth musclederived atherosclerotic plaque cells. Nat. Commun. 9, 2073 (2018).

40. Fadous-Khalife, M. C. et al. Kruppel-like factor 4: a new potential biomarker of lung cancer. Mol. Clin. Oncol. 5, 35-40 (2016).

41. Kim, K. K., Sheppard, D. \& Chapman, H. A. TGF-betal signaling and tissue fibrosis. Cold Spring Harb. Perspect. Biol. 10, a022293 (2018).

42. Meng, X. M., Nikolic-Paterson, D. J. \& Lan, H. Y. TGF-beta: the master regulator of fibrosis. Nat. Rev. Nephrol. 12, 325-338 (2016).

43. Klinkhammer, B. M., Floege, J. \& Boor, P. PDGF in organ fibrosis. Mol. Asp. Med. 62, 44-62 (2018).

44. Fiore, V. F. et al. Alphavbeta3 Integrin drives fibroblast contraction and strain stiffening of soft provisional matrix during progressive fibrosis. JCI Insight $\mathbf{3}$, e97597 (2018)

45. Atamas, S. P. Complex cytokine regulation of tissue fibrosis. Life Sci. 72, 631-643 (2002).

46. Curciarello, R., Docena, G. H. \& MacDonald, T. T. The role of cytokines in the fibrotic responses in Crohn's disease. Front. Med. 4, 126 (2017).

47. Deshmane, S. L., Kremlev, S., Amini, S. \& Sawaya, B. E. Monocyte chemoattractant protein-1 (MCP-1): an overview. J. Interferon Cytokine Res. 29, 313-326 (2009).

48. Inoshima, I. et al. Anti-monocyte chemoattractant protein-1 gene therapy attenuates pulmonary fibrosis in mice. Am. J. Physiol. Lung Cell Mol. Physiol. 286, L1038-1044 (2004).

49. Sun, L. et al. New concepts of IL-10-induced lung fibrosis: fibrocyte recruitment and M2 activation in a CCL2/CCR2 axis. Am. J. Physiol. Lung Cell Mol. Physiol. 300, L341-353 (2011). 
50. Kurahashi, T. et al. Forkhead box M1 transcription factor drives liver inflammation linking to hepatocarcinogenesis in mice. Cell Mol. Gastroenterol. Hepatol. 9, 425-446 (2020).

51. Penke, L. R. et al. FOXM1 is a critical driver of lung fibroblast activation and fibrogenesis. J. Clin. Investig. 128, 2389-2405 (2018).

52. Li, Q. et al. Disruption of Klf4 in villin-positive gastric progenitor cells promotes formation and progression of tumors of the antrum in mice. Gastroenterology 142, 531-542 (2012).

53. Kim, K. K. et al. Alveolar epithelial cell mesenchymal transition develops in vivo during pulmonary fibrosis and is regulated by the extracellular matrix. Proc. Natl Acad. Sci. USA 103, 13180-13185 (2006).

54. Li, R. et al. Pdgfra marks a cellular lineage with distinct contributions to myofibroblasts in lung maturation and injury response. Elife 7, e36865 (2018).

55. Xie, T. et al. Transcription factor TBX4 regulates myofibroblast accumulation and lung fibrosis. J. Clin. Investig. 126, 3626 (2016).

56. Lu, S. et al. Smooth muscle-derived progenitor cell myofibroblast differentiation through KLF4 downregulation promotes arterial remodeling and fibrosis. JCI Insight 5, e139445 (2020).

57. Sheikh, A. Q., Saddouk, F. Z., Ntokou, A., Mazurek, R. \& Greif, D. M. Cell autonomous and non-cell autonomous regulation of SMC progenitors in pulmonary hypertension. Cell Rep. 23, 1152-1165 (2018).

58. Sava, P. et al. Human pericytes adopt myofibroblast properties in the microenvironment of the IPF lung. JCI Insight 2, e96352 (2017).

59. Dave, J. M., Mirabella, T., Weatherbee, S. D. \& Greif, D. M. Pericyte ALK5/ TIMP3 axis contributes to endothelial morphogenesis in the developing brain. Dev. Cell 44, 665-678 e666 (2018)

60. Zhang, K., Gharaee-Kermani, M., Jones, M. L., Warren, J. S. \& Phan, S. H. Lung monocyte chemoattractant protein-1 gene expression in bleomycininduced pulmonary fibrosis. J. Immunol. 153, 4733-4741 (1994).

61. Singer, C. A., Salinthone, S., Baker, K. J. \& Gerthoffer, W. T. Synthesis of immune modulators by smooth muscles. Bioessays 26, 646-655 (2004).

62. Lim, S. \& Park, S. Role of vascular smooth muscle cell in the inflammation of atherosclerosis. BMB Rep. 47, 1-7 (2014).

63. Moore, B. B. et al. Protection from pulmonary fibrosis in the absence of CCR2 signaling. J. Immunol. 167, 4368-4377 (2001).

64. Raghu, G. et al. CC-chemokine ligand 2 inhibition in idiopathic pulmonary fibrosis: a phase 2 trial of carlumab. Eur. Respir. J. 46, 1740-1750 (2015).

65. Madisen, L. et al. A robust and high-throughput Cre reporting and characterization system for the whole mouse brain. Nat. Neurosci. 13, 133-140 (2010).

66. Muzumdar, M. D., Tasic, B., Miyamichi, K., Li, L. \& Luo, L. A global doublefluorescent Cre reporter mouse. Genesis 45, 593-605 (2007).

67. Srinivas, S. et al. Cre reporter strains produced by targeted insertion of EYFP and ECFP into the ROSA26 locus. BMC Dev. Biol. 1, 4 (2001).

68. Katz, J. P. et al. Loss of Klf4 in mice causes altered proliferation and differentiation and precancerous changes in the adult stomach. Gastroenterology 128, 935-945 (2005).

69. Wendling, O., Bornert, J. M., Chambon, P. \& Metzger, D. Efficient temporallycontrolled targeted mutagenesis in smooth muscle cells of the adult mouse. Genesis 47, 14-18 (2009).

70. Chen, Q. et al. Endothelial cells are progenitors of cardiac pericytes and vascular smooth muscle cells. Nat. Commun. 7, 12422 (2016).

\section{Acknowledgements}

The authors thank Greif Laboratory members for input and Aglaia Ntokou and Giorgio Mottola for technical experimental help. The authors also thank I. Weissman, D. Metzger and P. Chambon for mouse strains. Funding was provided by the Department of Army (W81XWH-18-1-0629 to D.M.G. and W81XWH-18-1-0630 to E.L.H.), National Institute of Health (R01HL125815, R01HL133016, R21NS088854 and R35HL150766 to D.M.G. and F32HL132532 to R.R.C.), and American Heart Association (Established Investigator Award, 19EIA34660321 to D.M.G.).

\section{Author contributions}

R.R.C., Y.X., E.G-V., A.Q.S., K.A.M., E.L.H. and D.M.G. conceived of and designed experiments, and R.R.C., Y.X., E.M.G., I.K. and A.Q.S. performed them. T.A. conducted scRNA-seq analysis, and N.K. provided infrastructure for this analysis. R.R.C. and R.G.-M. performed bioinformatic analyses. R.R.C., I.K., A.Q.S., E.L.H. and D.M.G. analyzed the results, and R.R.C., A.Q.S. and D.M.G. prepared the figures. R.R.C., Y.X., E.L.H. and D.M.G. wrote the manuscript. All authors reviewed and provided input on manuscript.

\section{Competing interests}

The authors declare no competing interests.

\section{Additional information}

Supplementary information The online version contains supplementary material available at https://doi.org/10.1038/s41467-021-27499-8.

Correspondence and requests for materials should be addressed to Daniel M. Greif.

Peer review information Nature Communications thanks the anonymous reviewers for their contribution to the peer review of this work.

Reprints and permission information is available at http://www.nature.com/reprints

Publisher's note Springer Nature remains neutral with regard to jurisdictional claims in published maps and institutional affiliations.

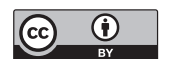

Open Access This article is licensed under a Creative Commons Attribution 4.0 International License, which permits use, sharing, adaptation, distribution and reproduction in any medium or format, as long as you give appropriate credit to the original author(s) and the source, provide a link to the Creative Commons license, and indicate if changes were made. The images or other third party material in this article are included in the article's Creative Commons license, unless indicated otherwise in a credit line to the material. If material is not included in the article's Creative Commons license and your intended use is not permitted by statutory regulation or exceeds the permitted use, you will need to obtain permission directly from the copyright holder. To view a copy of this license, visit http://creativecommons.org/ licenses/by/4.0/.

(C) The Author(s) 2021 